\title{
Index Coding Capacity: How Far Can One Go With Only Shannon Inequalities?
}

\author{
Hua Sun, Student Member, IEEE, and Syed Ali Jafar, Fellow, IEEE
}

\begin{abstract}
An interference alignment perspective is used to identify the simplest instances (minimum possible number of edges in the alignment graph, not more than 2 interfering messages at any destination) of index coding problems where non-Shannon information inequalities are necessary for capacity characterization. In particular, this includes the first known example of a multiple unicast (one destination per message) index coding problem where non-Shannon information inequalities are shown to be necessary. The simplest multiple unicast example has 7 edges in the alignment graph and 11 messages. The simplest multiple groupcast (multiple destinations per message) example has 6 edges in the alignment graph, 6 messages, and 10 receivers. For both the simplest multiple unicast and multiple groupcast instances, the best outer bound based on only Shannon inequalities is $2 / 5$, which is tightened to $11 / 28$ by the use of the Zhang-Yeung non-Shannon type information inequality, and the linear capacity is shown to be $5 / 13$ using the Ingleton inequality. Conversely, identifying the minimal challenging aspects of the index coding problem allows an expansion of the class of solved index coding problems up to (but not including) these instances.
\end{abstract}

Index Terms - Capacity, index coding, interference alignment, non-shannon inequalities.

\section{INTRODUCTION}

$\mathbf{T}$ HE capacity of a general "Index Coding" communication network is one of the most intriguing problems in network information theory. The index coding problem is simple to describe (only one link of finite capacity) but difficult to solve (remains open in general), it is the original setting for interference alignment [1], [2] but is only starting to be explored from an interference alignment perspective [3], [4], and while it is a network coding problem itself, it has been shown to be representative of all (including non-linear) network coding instances [5], [6]. The index coding problem has also been shown recently to be essentially equivalent (up to linear solutions) to the so called topological interference management problem [3], where the degrees of freedom of a partially connected wireless interference network or the capacity of a partially connected

Manuscript received March 27, 2013; revised February 2, 2014; accepted March 15, 2015. Date of publication April 6, 2015; date of current version May 15, 2015. This work was supported in part by ONR and in part by NSF under Award CCF-1317351 and Award CCF-1319104.

The authors are with the Center for Pervasive Communications and Computing, Department of Electrical Engineering and Computer Science, University of California at Irvine, Irvine, CA 92697 USA (e-mail: huas2@uci.edu; syed@uci.edu).

Communicated by A. Lozano, Associate Editor for Communications.

Color versions of one or more of the figures in this paper are available online at http://ieeexplore.ieee.org.

Digital Object Identifier 10.1109/TIT.2015.2418289 wired network are investigated with only a knowledge of the network topology available to the transmitters. As such the index coding problem presents an opportunity to tackle some of the fundamental challenges lying at the intersection of several open problems in network information theory.

\section{A. Prior Work}

Since its introduction in 1998 [1], many interesting instances of the index coding problem have been studied from coding theoretic, graph theoretic, and information theoretic perspectives, leading primarily to a variety of inner bounds (achievable schemes). The earliest inner bound, obtained by Birk and Kol and Bar-Yossef et al. in [1] and [7], is the clique cover of an index coding side information graph. The clique cover and its standard LP generalization, the fractional clique cover, correspond to orthogonal scheduling (analogous to TDMA/FDMA) in the parlance of interference networks [3] — only non-interfering groups of users are simultaneously scheduled for transmission. A linear programming inner bound is introduced by Blasiak et al. in [8], based on higher order submodularity and coincides with fractional hyperclique-cover number (reduces to fractional clique-cover number for multiple unicast instances). The fractional clique cover inner bound is generalized to a partition multicast inner bound by Tehrani et al. in [9]. The partition multicast approach corresponds to CDMA in interference networks - pseudo-random precoding sequences are used with the length of the sequences chosen to be just enough to provide each receiver enough equations so it can resolve all symbols from the transmissions that it can hear concurrently with its desired transmission [3]. The local chromatic number is proposed as an inner bound by Shanmugam et al. in [10] based on viewing the index coding problem as a vector assignment problem. This is equivalent to a restricted form of interference alignment, sometimes known as one-to-one alignment (as opposed to the more general concept of subspace alignment). For index coding problems that correspond to undirected graphs (equivalently, bidirected graphs) all of the inner bounds mentioned above the fractional clique cover, partition multicast, the linear programming bound based on higher-order submodularity, and the local chromatic number - are equivalent. They are also generally suboptimal. A family of undirected graph based index coding problems with $n$ nodes is presented by Blasiak et al. in [8] where the index coding capacity is $1 / 3$, but the best rate achievable by all inner bounds mentioned above is no more than $O\left(\frac{1}{n^{1 / 4}}\right)$. The inner bound that is 
tight for this class of index coding problems is given by the min-rank function, which was originally introduced by Yossef et al. in [7] and corresponds to the optimal scalar linear solution to the index coding problem. However, even the min-rank bound is known to be suboptimal. First, it corresponds to scalar linear coding capacity, which is generally outperformed by vector linear coding [5], [11]. Second, while the min-rank bound can be extended as shown in [12, Sec 4.10] to find the best vector linear coding solution for a given number of symbols per message, it has also been shown by Blasiak et al. and Rouayheb et al. for multiple groupcast index coding problems [5], [13], and by Maleki et al. for multiple unicast index coding problems [4], that the best linear schemes are still not optimal in general because they can be outperformed by non-linear schemes for certain instances of the index coding problem. Therefore, none of the inner bounds discussed so far has the potential to be generally optimal for the index coding problem.

\section{B. Recent Progress: Random Coding and Interference Alignment Approaches}

Two recently proposed approaches, while still in their infancy, offer new hope by bringing in new machinery to attack the index coding problem. These are the random coding approach by Arbabjolfaei et al. in [14], and the interference alignment perspective of Jafar and Maleki et al. in [3] and [4]. Random coding has been a universal ingredient of capacity optimal schemes, and therefore presents a potentially powerful "hammer" to the "nail" of index coding [14]. The random coding approach of [14] has so far settled the capacity region for all instances of the multiple unicast index coding problem with 5 or fewer messages. ${ }^{1}$ The interference alignment perspective presents a different kind of hammer (or perhaps a "jack knife" since it takes a finer view of the problem [14]), that has been extremely successful in wireless networks where a variety of interference alignment schemes, both linear and non-linear, have been developed to obtain degrees of freedom characterizations [2]. In addition to symmetric instances of the index coding problem such as neighboring antidotes, neighboring interferers, $X$ networks, and cellular topologies motivated by the topological interference management problem [4], the interference alignment perspective has so far settled the symmetric capacity of the class of multiple groupcast (which includes unicast as a special case) index coding problems where each alignment set has either no cycles or no forks [3].

Admittedly, the accomplishments of either approach thus far are infinitesimal relative to the full scope of the general index coding problem. However, the full strength of neither the random coding approach nor the interference alignment approach has yet been exhausted. Indeed it is apparent that both approaches have plenty of room to expand through capacity characterizations of increasingly broader classes of the index coding problem. Continued efforts towards such

\footnotetext{
${ }^{1}$ The capacity of an interesting sub-class of multiple unicast index coding problems with 6 messages, corresponding to the topologies that can arise in a 6-cell network, is settled by Naderializadeh and Avestimehr in [15].
}

expansions are therefore well motivated. At the same time, it is also important to understand the limitations of these approaches by identifying the challenges that lie ahead. To this end, we note that all index coding capacity results obtained so far from random coding and interference alignment perspectives, have relied on only Shannon inequalities for the outer bounds. One indication of a substantial challenge could be the necessity of non-Shannon inequalities. Remarkably, for multiple unicast index coding problems it is not known whether non-polymatroidal (non-Shannon, Ingleton) inequalities are ever necessary. Therefore, we would like to find out if instances of multiple unicast index coding exist where Shannon inequalities do not suffice, and if so, then we would like to identify the simplest possible such instance. The emphasis on simplicity is important for the challenging aspects to be as broadly relevant as possible.

\section{Non-Shannon Inequalities}

To characterize the information-theoretic/linear capacity of communication networks, it is important to understand the fundamental limitations of entropy/vector spaces, in the form of information inequalities/linear rank inequalities. Since linear coding schemes are only a special case of all possible coding schemes, information inequalities are a proper subset of linear rank inequalities. It is well known that both entropy space and vector space satisfy the basic polymatroidal axioms, which is equivalent to the non-negativeness of Shannon information measurements, also known as Shannon-type information inequalities or basic inequalities [16]. With up to 3 random variables/subspaces, all the information inequalities/linear rank inequalities coincide with the polymatroidal axioms [17]. However, when the number of elements increases to 4 , both information inequalities and linear rank inequalities involve additional constraints beyond the polymatroidal axioms. For information inequalities, the first non-Shannon-type information inequality with 4 random variables was discovered in 1998 by Zhang and Yeung [18], followed by many others, see [19]-[21]. It is shown by Matus that even with only 4 random variables, the list of non-Shannon-type information inequalities is infinite [20]. For linear rank inequalities, Ingleton [22] showed a set of inequalities that is not implied by basic inequalities, but when combined with them, constitutes a complete characterization of all linear rank inequalities that must be satisfied by 4 subspaces of a vector space [17]. However, Ingleton inequalities are still not enough to go beyond 4 subspaces [23], [24]. Recent work finds all the linear rank inequalities for 5 subspaces (there are 24 such inequalities in addition to the Shannon and Ingleton inequalities), while cases with more than 5 subspaces are still open [24].

All these mathematical inequalities have found their usage and correspondence in network coding studies [25], [26]. Dougherty et al. in [27] have shown that Shannon inequalities are not always sufficient for the general network coding problem. For index coding problems the insufficiency of Shannon inequalities is established by Blasiak et al. in [8], albeit only in the context of multiple groupcast index coding 
where each message is desired by multiple receivers. For multiple unicast index coding, however, no example is known that shows that Shannon inequalities are insufficient. For instance, the general outer bound for multiple unicast index coding presented in [14, Th. 1] is based directly on Shannon inequalities and it is noted afterwards that it is not known whether the outer bound is tight in general. It is also notable that this bound is found to be tight for all instances of multiple unicast index coding with 5 or fewer messages. For further details about information inequalities and linear rank inequalities, we refer to the excellent tutorials in [28], [29], and references therein.

Since the notation and definitions used in this work are the same as in [3], we proceed directly to the results. The relevant definitions from [3] are summarized for the sake of completeness in Appendix.

\section{RESULTS}

\section{A. Criteria for the Simplest Example}

Our first goal is to prove that Shannon inequalities are insufficient even for multiple unicast index coding, so that the outer bound in [14, Th. 1] cannot be tight in general. For such a result, the simplest example is the most powerful. Therefore, we would like to find an example that involves only those features that would make it a part of any interesting class of index coding problems. For instance, a multiple unicast example would prove the insufficiency result for both multiple unicast and the multiple groupcast settings, because multiple unicast settings are contained within the class of multiple groupcast settings. So our example must be a multiple unicast setting. This is especially critical because the insufficiency of Shannon inequalities is already shown for the more general groupcast setting [8].

Continuing the thought, even within the class of multiple unicast index coding problems, we would like to identify the simplest example possible, for the result to hold as broadly as possible. The idea of 'simplicity' can be quite subjective. However, coming from an interference alignment perspective, we find it natural to interpret it in terms of the 'type' and 'number' of edges in the alignment graph, as defined in [3].

The type of an alignment edge refers to whether the alignments that it demands ${ }^{2}$ are one-to-one alignments or subspace-alignments. Let us elaborate on this distinction. Consider an interference network where a receiver experiences interference from only two undesired messages. The principle of interference alignment dictates that these two undesired transmissions should try to collectively occupy as small a signal space as possible. Since there are only two interferers, the only way to consolidate the interference space is for them to align with each other as much as possible. So what is demanded is a one-to-one alignment. Now, consider a different scenario where the receiver sees interference from

\footnotetext{
${ }^{2}$ It is important to distinguish between an alignment demand, which represents an edge in the alignment graph, and an alignment solution which could be the optimal vector space assignment. For example, even if all interferencealignment demands are one-to-one, the optimal solution may require subspace alignments strictly beyond one-to-one alignments. Such examples are not uncommon, e.g., one appears in Fig. 6 of this paper.
}

three or more interferers. The principle of interference alignment again dictates that these three or more undesired transmissions should try to collectively occupy as small a signal space as possible. Note however, that a direct alignment of any interferer with any other interferer is no longer the only way to consolidate the signals. For instance, one interferer may align itself in the space spanned jointly by the others without even partially aligning with any of them on a one-to-one basis. What is demanded here is the more general notion of subspace-alignment. Since subspace-alignment includes one-to-one alignment as a special case, we naturally require that the simplest example should have only one-to-one alignment demands, i.e., no receiver should see more than two interferers.

The number of alignment conditions simply refers to the number of edges in the alignment graph. Since each edge represents a desired alignment, the number of edges roughly corresponds to the number of dependencies among the variables involved. By this understanding, the simplest example is the one with the minimum number of dependencies, i.e., fewest edges in the alignment graph. At this point we have identified the criteria that the simplest example should satisfy.

Criteria for the Simplest Example:

1) Requires non-Shannon inequalities.

2) Is a multiple unicast index coding problem.

3) Each receiver sees no more than 2 interferers (one-to-one alignment demands).

4) Has the minimum number of edges in its alignment graph (among all examples that satisfy the first three criteria).

Remark: While this definition of simplicity is motivated by the interference alignment perspective, other definitions may be interesting from other perspectives. For example, since the approach taken by [14] involves solving all multiple unicast index coding problems up to a given number of users, the simplest example in that sense might be the one with the minimum number of users, disregarding the number of edges in the alignment graph and the restriction to one-to-one alignments. Progress towards identifying the simplest example in this sense (with further restriction to symmetric index coding problems represented by bidirectional side information graphs) is reported by Baber et al. in [30].

Note that it was not known a priori that an example satisfying our criteria even exists. However, identifying these criteria helps us search for such an example. Our initial motivation in performing this search was to either find the simplest such example, or to settle the capacity for this entire class of index coding problems. What is remarkable is that in all instances that belong to this class, for which the capacity was previously known, the capacity coincided with the internal conflict bound [3, Th. 6], which is based only on Shannon inequalities. Specifically, in all such cases that were previously solved, the symmetric capacity was equal to $\frac{\Delta}{2 \Delta+1}$, where $\Delta$ is the minimum internal conflict distance of the alignment graph [3]. Based on this observation, our initial expectation had been that the conflict bound may be always tight for this class of problems, and therefore Shannon inequalities will be sufficient. However, the result turns out to 


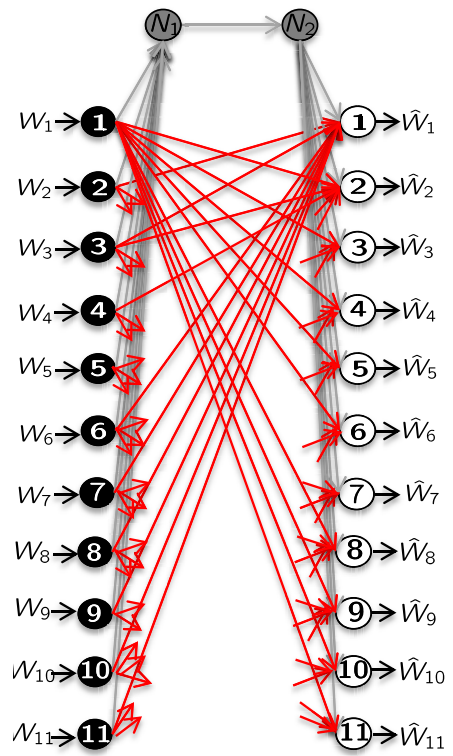

(a)

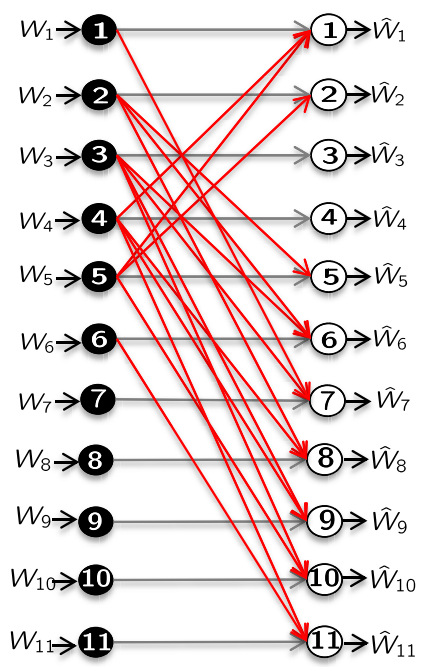

(b)
Interference at

each destination

$\left\{W_{4}, W_{5}\right\}$

$\left\{W_{5}\right\}$

$\{\emptyset\}$

$\{\emptyset\}$

$\left\{W_{2}\right\}$

$\left\{W_{2}, W_{3}\right\}$

$\left\{W_{1}, W_{3}\right\}$

$\left\{W_{2}, W_{4}\right\}$

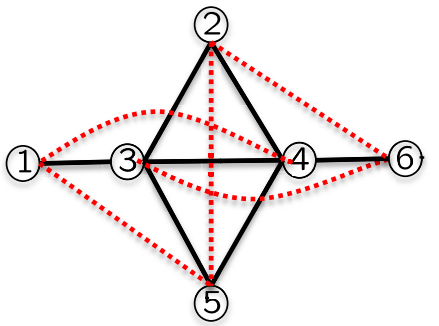

(7)

Fig. 1. The Simplest Example of a Multiple Unicast Index Coding Problem where Non-Shannon Inequalities are Necessary. (a) Red links represent antidotes (not all are shown), and (b) Red links show all the interference links (complements of the antidote links), and (c) Interfering messages (missing antidotes) at each destination are listed, and (d) Alignment graph (solid black edges) and conflict graph (dashed red edges). Only internal conflicts are shown.

be somewhat unexpected. The conflict bound is indeed found to be tight for all networks in this class as long as there are no overlapping cycles in the alignment graph, i.e., cycles that share an edge. However, once we involve overlapping cycles we almost immediately run into the simplest example where non-Shannon inequalities are necessary.

\section{B. The Simplest Example Where Non-Shannon Inequalities Are Necessary}

1) Construction: The simplest example where non-Shannon inequalities are necessary, is shown in Fig. 1. It is a multiple unicast setting with 11 messages, in which no receiver sees more than 2 interferers (only one-to-one alignment demands). Since the antidote graph in Fig. 1(a) has too many edges, the interference graph, which is the complement of the antidote graph, and is therefore quite sparsely connected, is shown in Fig. 1(b) instead. The interfering messages at each receiver (equivalently, the antidotes missing at each receiver) are also listed in Fig. 1(c) for convenience.

From an interference alignment perspective, the essence of the problem is captured by its alignment graph, shown in Fig. 1(d) with solid black edges. The alignment graph contains a node for each message, and each edge connects two interferers that are seen by the same destination, i.e., the pairs that are listed in Fig. 1(c). Note the remarkable simplicity of the alignment graph, which involves only 7 alignment edges (solid black edges). As will be evident soon enough, the core of the problem, which makes non-Shannon inequalities necessary, has to do mainly with the four messages, $W_{2}, W_{3}, W_{4}, W_{5}$, that comprise the inner diamond. Messages $W_{1}, W_{6}$ create the requisite internal conflicts for the inner diamond to set off non-Shannon inequalities. The remaining messages, $W_{7}, W_{8}, W_{9}, W_{10}, W_{11}$, play only a marginal supporting role by demanding the alignments that bring the inner diamond into being.

2) Necessity of Non-Shannon Inequalities: For the tightest Shannon-type outer bound on the index coding capacity region, i.e., based on sub-modularity, we refer to [14, Th. 1], which we re-state here in an equivalent form that is more conducive for our purpose. We start by introducing the relevant notation.

For unicast problems, there are $N$ sources and $N$ destinations. Source $j$ has a desired message $W_{j}$ for destination $j, j \in[1: N] . \mathcal{A}_{j}$ is the index set of antidotes available at destination $j$. Define $\mathcal{T}$ to be the set of all real-valued functions $T_{\mathcal{J}}, \mathcal{J} \subseteq[1: N]$, such that

1) $T_{\varnothing}=0$,

2) $T_{[1: N]}=1$,

3) $T_{\mathcal{J}} \leq T_{\mathcal{K}}$ for all $\mathcal{J} \subseteq \mathcal{K} \subseteq[1: N]$, and

4) $T_{\mathcal{J} \cap \mathcal{K}}+T_{\mathcal{J} \cup \mathcal{K}} \leq T_{\mathcal{J}}+T_{\mathcal{K}}$ for all $\mathcal{J}, \mathcal{K} \subseteq[1: N]$.

We will refer to $\mathcal{T}$ as the set of all sub-modular functions. The tightest Shannon-type outer bound presented in [14, Th. 1] can then be equivalently re-stated as follows.

Theorem 1 (Arbabjolfaei et al.): Let $\mathcal{B}_{j}=[1: N] \backslash\left(\{j\} \cup \mathcal{A}_{j}\right)$ be the index set of interfering messages at destination $j$. The capacity region, $\mathcal{C}$, of a multiple-unicast index coding problem is bounded as follows.

$$
\begin{aligned}
\mathcal{C} \subseteq \mathcal{C}_{o} \triangleq \bigcup_{T \in \mathcal{T}}\left\{\left(R_{1}, R_{2}, \cdots, R_{N}\right)\right. & \text { such that } \left.R_{j} \leq T_{\{j\} \cup \mathcal{B}_{j}}-T_{\mathcal{B}_{j}}, \quad j \in[1: N]\right\}
\end{aligned}
$$

Define $C_{o}$ to be the tightest outer bound on the symmetric capacity $C$, that is implied by Theorem 1 for the index coding instance of Fig. 1.

$$
C \leq C_{o} \triangleq \max _{(R, R, \cdots, R) \in \mathcal{C}_{o}} R
$$


The tightest outer bound, $C_{o}$, for the index coding instance of Fig. 1, based only on Shannon inequalities, is explicitly obtained in Theorem 2.

Theorem 2: $C_{o}=\frac{2}{5}$.

The proof is presented in Section IV-B, and consists of two parts where we prove that $C_{o} \leq \frac{2}{5}$ and $C_{o} \geq \frac{2}{5}$. The former follows from the internal conflict bound of [3] which is based only on Shannon inequalities. The latter is shown by an explicit construction of a sub-modular $T$ function that allows the symmetric rate $2 / 5$, thus implying that Shannon-type inequalities cannot rule out the symmetric rate vector $\left(R_{1}, R_{2}, \cdots, R_{N}\right)=\left(\frac{2}{5}, \frac{2}{5}, \cdots, \frac{2}{5}\right)$.

Finally, the necessity of non-Shannon inequalities is established in the following theorem. It is shown that Zhang-Yeung non-Shannon-type information inequality [18] yields a tighter outer bound than $C_{o}$.

Theorem 3: For the index coding instance of Fig. 1, the Zhang-Yeung non-Shannon-type information inequality yields an outer bound value of $\frac{11}{28}$, which is smaller than $\frac{2}{5}$.

The proof is presented in Section IV-C, and consists of two parts. The first part uses the alignment chain $W_{1}-W_{3}-W_{4,5}$ (symmetrically $W_{6}-W_{4}-W_{2,3}$ ) to obtain a lower bound on the dimensions occupied by the entropic space of the triangle comprised of $W_{2}, W_{3}, W_{4}\left(W_{3}, W_{4}, W_{5}\right)$. This part only involves applying submodularity, i.e., Shannon-type information inequalities. The second part deals with the diamond comprised of $W_{2}, W_{3}, W_{4}, W_{5}$. Here we use the Zhang-Yeung non-Shannon-type information inequality to obtain an upper bound for the dimensions occupied by the entropic space of the two triangles comprised of $W_{2}, W_{3}, W_{4}$ and $W_{3}, W_{4}, W_{5}$. Combining these two pieces yields the desired outer bound.

3) Vector Space Interpretation and Linear Capacity: Let us explore from a vector space perspective why we need a non-polymatroidal inequality. Why do polymatroidal axioms not allow an outer bound smaller than $\frac{2}{5}$ ? Let us associate with each message $W_{i}$, the vector space $\mathbf{V}_{i}$. We will denote the vector space spanned by the union and intersection of spans of $\mathbf{V}_{i}$ and $\mathbf{V}_{j}$ as $\left(\mathbf{V}_{i}, \mathbf{V}_{j}\right)$ and $\left(\mathbf{V}_{i} \cap \mathbf{V}_{j}\right)$, respectively. Suppose we assign (normalized) subspace dimensions as $\operatorname{dim}\left(\mathbf{V}_{2}\right)=\operatorname{dim}\left(\mathbf{V}_{3}\right)=\operatorname{dim}\left(\mathbf{V}_{4}\right)=\operatorname{dim}\left(\mathbf{V}_{5}\right)=\frac{2}{5}$, $\operatorname{dim}\left(\mathbf{V}_{2}, \mathbf{V}_{3}\right)=\operatorname{dim}\left(\mathbf{V}_{2}, \mathbf{V}_{4}\right)=\operatorname{dim}\left(\mathbf{V}_{3}, \mathbf{V}_{4}\right)=$ $\operatorname{dim}\left(\mathbf{V}_{3}, \mathbf{V}_{5}\right)=\operatorname{dim}\left(\mathbf{V}_{4}, \mathbf{V}_{5}\right)=\frac{3}{5}$, and $\operatorname{dim}\left(\mathbf{V}_{2}, \mathbf{V}_{5}\right)=$ $\operatorname{dim}\left(\mathbf{V}_{2}\right)+\operatorname{dim}\left(\mathbf{V}_{5}\right)=\frac{4}{5}$ (because $W_{2}$ and $W_{5}$ conflict with each other, i.e., they cannot align) and finally, for the two triangles that create the diamond shape in the alignment graph, $\operatorname{dim}\left(\mathbf{V}_{2}, \mathbf{V}_{3}, \mathbf{V}_{4}\right)=\operatorname{dim}\left(\mathbf{V}_{3}, \mathbf{V}_{4}, \mathbf{V}_{5}\right)=\frac{4}{5}$. It is easy to verify that this dimension allocation satisfies all submodularity constraints, so an outer bound (for linear capacity) smaller than $\frac{2}{5}$ is not possible through polymatroidal axioms (submodularity) alone. Now let us see why the submodularity bound must be loose, i.e., why the given dimension allocation cannot be satisfied by any vector space assignment. Because $\left(\mathbf{V}_{3}, \mathbf{V}_{4}\right)$ occupies $\frac{3}{5}$ dimensions, for the vector space $\left(\mathbf{V}_{2}, \mathbf{V}_{3}, \mathbf{V}_{4}\right)$ to occupy $\frac{4}{5}$ dimensions, $\mathbf{V}_{2}$ must have $\frac{1}{5}$ new dimensions that have no intersection with $\left(\mathbf{V}_{3}, \mathbf{V}_{4}\right)$. So it has only $\frac{1}{5}$ remaining dimensions that can intersect with $\left(\mathbf{V}_{3}, \mathbf{V}_{4}\right)$. But it needs to intersect with each of $\mathbf{V}_{3}$ and $\mathbf{V}_{4}$ individually in $\frac{2}{5}+\frac{2}{5}-\frac{3}{5}=\frac{1}{5}$ dimensions. Therefore $\mathbf{V}_{2}$ must intersect with

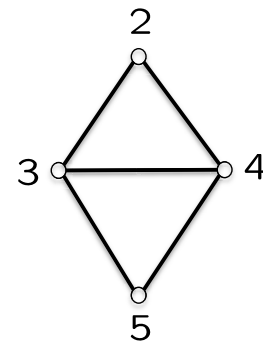

(a)

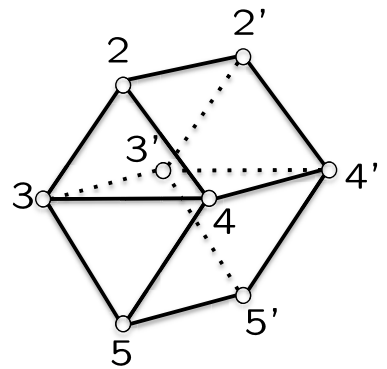

(b)
Fig. 2. (a) Diamond part of the alignment graph and (b) Vamos matroid.

$\mathbf{V}_{3}$ in the same $\frac{1}{5}$ dimensional space within which it intersects with $\mathbf{V}_{4}$. Therefore, the intersecting space of $\mathbf{V}_{2}$ with $\left(\mathbf{V}_{3}, \mathbf{V}_{4}\right)$ must be the same as the intersecting space of $\mathbf{V}_{3}$ with $\mathbf{V}_{4}$, i.e., $\left(\mathbf{V}_{2} \cap\left(\mathbf{V}_{3}, \mathbf{V}_{4}\right)\right)=\left(\mathbf{V}_{2} \cap \mathbf{V}_{3}\right)=\left(\mathbf{V}_{2} \cap \mathbf{V}_{4}\right)=\left(\mathbf{V}_{3} \cap \mathbf{V}_{4}\right)$. The same arguments can be made for the other triangle as well, i.e., $\left(\mathbf{V}_{5} \cap\left(\mathbf{V}_{3}, \mathbf{V}_{4}\right)\right)=\left(\mathbf{V}_{5} \cap \mathbf{V}_{3}\right)=\left(\mathbf{V}_{5} \cap \mathbf{V}_{4}\right)=\left(\mathbf{V}_{3} \cap \mathbf{V}_{4}\right)$. However, this means that $\mathbf{V}_{2}$ and $\mathbf{V}_{5}$ must intersect in these dimensions as well. But this is a contradiction because $W_{2}$ conflicts with $W_{5}$.

As mentioned earlier, this contradiction cannot be captured by polymatroidal inequalities alone. However, the contradiction can be obtained as follows, which is shown at the top of the next page. For clarity, we include a derivation of $(*)$ in Section IV-A.

Note that in (3), the five terms on the left hand side correspond to the five edges of the diamond in Fig. 2(a). Every step in this derivation is generally applicable to arbitrary vector subspaces $\mathbf{V}_{2}, \mathbf{V}_{3}, \mathbf{V}_{4}, \mathbf{V}_{5}$. In fact, what we have derived in (3) is precisely the Ingleton inequality, which must be satisfied by any four vector subspaces. Plugging in the given dimension allocations we have on the left hand side a value of $\frac{3}{5} \times 5=3$ and on the right hand side a value of $\frac{2}{5}+\frac{2}{5}+\frac{4}{5}+\frac{4}{5}+\frac{4}{5}=\frac{16}{5}>3$, which violates (3), thus producing a contradiction.

While we arrived at this example from an interference alignment perspective, there are curious parallels to the Vamos matroid, previously used to establish the necessity of non-Shannon inequalities in the general network coding problem [27] and in the multiple groupcast index coding problem [8]. Even the core of the alignment graph bears a resemblance to the Vamos matroid, as illustrated in Fig. 2.

The Vamos matroid is an eight element $\left(\left\{2,2^{\prime}, 3,3^{\prime}, 4,4^{\prime}, 5,5^{\prime}\right\}\right)$ matroid with rank 4. All 4-element subsets are independent (naturally with rank 4) except the five planes $\left\{2,2^{\prime}, 3,3^{\prime}\right\},\left\{2,2^{\prime}, 4,4^{\prime}\right\},\left\{3,3^{\prime}, 4,4^{\prime}\right\},\left\{3,3^{\prime}, 5,5^{\prime}\right\}$, $\left\{4,4^{\prime}, 5,5^{\prime}\right\}$, which have rank 3 each. Now if we establish a correspondence $\mathbf{V}_{2} \equiv\left\{2,2^{\prime}\right\}, \mathbf{V}_{3} \equiv\left\{3,3^{\prime}\right\}, \mathbf{V}_{4} \equiv\left\{4,4,^{\prime}\right\}$ and $\mathbf{V}_{5} \equiv\left\{5,5^{\prime}\right\}$ and a normalization so that rank 1 in the matroid is mapped to $\frac{1}{5}$ vector space dimensions, we find the dimension allocation that assigns $\frac{2}{5}$ dimensions per message for the diamond alignment graph matches the rank function of the Vamos matroid. Now, it is well known that the Vamos matroid is not representable (realizable by vector spaces) [31] and also not representable by entropic spaces, making this a natural example to require the use of 


$$
\begin{aligned}
& \operatorname{dim}\left(\mathbf{V}_{2} \cap \mathbf{V}_{5}\right) \geq \operatorname{dim}\left(\mathbf{V}_{2} \cap\left(\mathbf{V}_{3} \cap \mathbf{V}_{4}\right)\right)+\operatorname{dim}\left(\mathbf{V}_{5} \cap\left(\mathbf{V}_{3} \cap \mathbf{V}_{4}\right)\right)-\operatorname{dim}\left(\mathbf{V}_{3} \cap \mathbf{V}_{4}\right) \\
& \geq \operatorname{dim}\left(\mathbf{V}_{2} \cap \mathbf{V}_{3}\right)+\operatorname{dim}\left(\mathbf{V}_{2} \cap \mathbf{V}_{4}\right)-\operatorname{dim}\left(\mathbf{V}_{2} \cap\left(\mathbf{V}_{3}, \mathbf{V}_{4}\right)\right) \\
&+\operatorname{dim}\left(\mathbf{V}_{5} \cap \mathbf{V}_{3}\right)+\operatorname{dim}\left(\mathbf{V}_{5} \cap \mathbf{V}_{4}\right)-\operatorname{dim}\left(\mathbf{V}_{5} \cap\left(\mathbf{V}_{3}, \mathbf{V}_{4}\right)\right)-\operatorname{dim}\left(\mathbf{V}_{3}, \mathbf{V}_{4}\right) \\
& \Rightarrow \operatorname{dim}\left(\mathbf{V}_{2}\right)+\operatorname{dim}\left(\mathbf{V}_{5}\right)-\operatorname{dim}\left(\mathbf{V}_{2}, \mathbf{V}_{5}\right) \geq \operatorname{dim}\left(\mathbf{V}_{2}\right)+\operatorname{dim}\left(\mathbf{V}_{3}\right)-\operatorname{dim}\left(\mathbf{V}_{2}, \mathbf{V}_{3}\right)+\operatorname{dim}\left(\mathbf{V}_{2}\right)+\operatorname{dim}\left(\mathbf{V}_{4}\right)-\operatorname{dim}\left(\mathbf{V}_{2}, \mathbf{V}_{4}\right) \\
& \quad-\operatorname{dim}\left(\mathbf{V}_{2}\right)-\operatorname{dim}\left(\mathbf{V}_{3}, \mathbf{V}_{4}\right)+\operatorname{dim}\left(\mathbf{V}_{2}, \mathbf{V}_{3}, \mathbf{V}_{4}\right)+\operatorname{dim}\left(\mathbf{V}_{5}\right)+\operatorname{dim}\left(\mathbf{V}_{3}\right)-\operatorname{dim}\left(\mathbf{V}_{5}, \mathbf{V}_{3}\right) \\
& \quad+\operatorname{dim}\left(\mathbf{V}_{5}\right)+\operatorname{dim}\left(\mathbf{V}_{4}\right)-\operatorname{dim}\left(\mathbf{V}_{5}, \mathbf{V}_{4}\right)-\operatorname{dim}\left(\mathbf{V}_{5}\right)-\operatorname{dim}\left(\mathbf{V}_{3}, \mathbf{V}_{4}\right)+\operatorname{dim}\left(\mathbf{V}_{3}, \mathbf{V}_{4}, \mathbf{V}_{5}\right)-\operatorname{dim}\left(\mathbf{V}_{3}, \mathbf{V}_{4}\right) \\
& \Rightarrow \operatorname{dim}\left(\mathbf{V}_{2}, \mathbf{V}_{3}\right)+\operatorname{dim}\left(\mathbf{V}_{2}, \mathbf{V}_{4}\right)+\operatorname{dim}\left(\mathbf{V}_{3}, \mathbf{V}_{4}\right)+\operatorname{dim}\left(\mathbf{V}_{3}, \mathbf{V}_{5}\right)+\operatorname{dim}\left(\mathbf{V}_{4}, \mathbf{V}_{5}\right) \\
& \geq \\
& \geq \operatorname{dim}\left(\mathbf{V}_{3}\right)+\operatorname{dim}\left(\mathbf{V}_{4}\right)+\operatorname{dim}\left(\mathbf{V}_{2}, \mathbf{V}_{5}\right)+\operatorname{dim}\left(\mathbf{V}_{2}, \mathbf{V}_{3}, \mathbf{V}_{4}\right)+\operatorname{dim}\left(\mathbf{V}_{3}, \mathbf{V}_{4}, \mathbf{V}_{5}\right)
\end{aligned}
$$

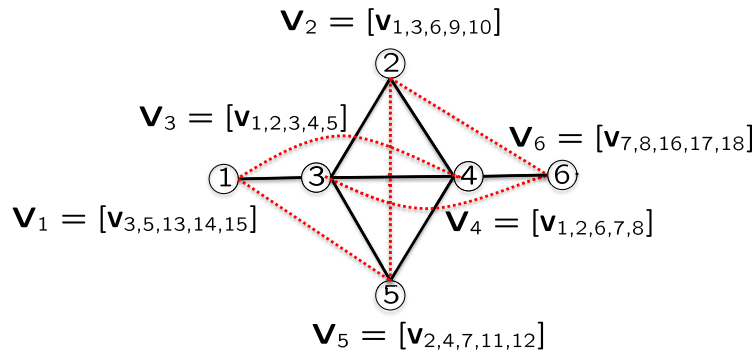

(7) $\mathbf{V}_{7}$

(9) $\mathbf{V}_{9}$

(10) $\mathbf{V}_{10}$

(11) $\mathbf{V}_{11}$

$\mathbf{V}_{5}=\left[\mathbf{v}_{2,4,7,11,12}\right]$

Fig. 3. A vector space assignment that avoids all conflicts. Symmetric rate achieved is $5 / 13$ per message. $\mathbf{v}_{i}, \mathbf{v}_{j}$ is abbreviated as $\mathbf{v}_{i, j}$, etc.

the Ingleton and non-Shannon inequalities. So, whether by coincidence or as a manifestation of a deeper mathematical property, in our simplest example motivated by interference alignment, the inner core of the alignment graph appears to be capturing the core dependence relationships of the Vamos matroid, with only marginal support from other parts of the alignment graph, instead of relying on all the circuits of the matroid. As a result, while the groupcast example based on the Vamos matroid used in [8] consists of 200 receiver nodes (the number of nodes associated with distinct circuits of the Vamos matroid), our unicast example involves only 11 receivers. On the other hand, because of the need for supporting messages our example does involve more messages (11 instead of 8). This is also because we want a unicast setting. As we will show later in this work, if we relax the problem to groupcast settings we can reduce the number of messages even further, to 6 .

The vector space bounds based on the Ingleton inequality lead us to the linear capacity of our simplest example network.

Theorem 4: The symmetric linear capacity for the index coding instance shown in Fig. 1 is $\frac{5}{13}$.

The outer bound follows as discussed previously, by the use of the Ingleton inequality which must be satisfied by all vector spaces. The proof is presented in Section IV-D. Here we give the achievable scheme. The achievable scheme is based on one-to-one alignment (see Fig. 3). The goal is to operate over 13 channel uses and choose 5 precoding vectors for each message, along which 5 symbols for that message will be sent. The design uses insights from the outer bound to decide how much alignment should occur between the signal spaces. Here we mention some of the key values to facilitate the understanding of alignment. Denote the precoding matrix formed by 5 precoding vectors for message $W_{i}$ as $\mathbf{V}_{i}$.
Then we have, $\operatorname{dim}\left(\mathbf{V}_{i}, \mathbf{V}_{j}\right)=\frac{8}{13}$ whenever there is a solid black edge between $i, j$ in the alignment graph, and $\operatorname{dim}\left(\mathbf{V}_{2} \cap \mathbf{V}_{3} \cap \mathbf{V}_{4}\right)=\operatorname{dim}\left(\mathbf{V}_{3} \cap \mathbf{V}_{4} \cap \mathbf{V}_{5}\right)=\frac{1}{13}$. This is accomplished as follows. Generate $18+5 \times 5=43$ vectors, each $13 \times 1$, that are in general position (any 13 of them are linearly independent), over a sufficiently large field. For $\mathbf{V}_{i}, i \in\{1,2, \ldots, 6\}$, assign the first 18 vectors according to Fig. 3. For $\mathbf{V}_{i}, i \in\{7,8, \ldots, 11\}$, assign 5 of the remaining vectors to each. It is easy to check all internal conflicts are avoided and the space occupied by each alignment edge is $\frac{8}{13}$, leaving enough space, $1-\frac{8}{13}=\frac{5}{13}$, for the desired signal. Then we can choose the receiving filter matrices to be the null space of their corresponding interference spaces and use the linear scheme described in detail in [3, Appendix A] to decode successfully.

4) There Is No Simpler Example: We now prove that this is indeed the simplest example where non-Shannon information inequalities are necessary. We will prove that for all multiple unicast index coding problems where each receiver is interfered by at most two messages (demanding only one-to-one alignments) and where the number of edges in the alignment graph is fewer than 7 , the symmetric capacity is given by the internal conflict bound, $\frac{\Delta}{2 \Delta+1}$, so that only Shannon-inequalities suffice. Half-rate-feasible networks are already solved in [3] and [8] through only Shannon inequalities, so we will concern ourselves with only half-rate-infeasible settings in the following theorem.

Theorem 5: For the class of half-rate-infeasible multipleunicast index coding problems where each destination is interfered by at most two messages, if each alignment set contains fewer than or equal to 6 alignment edges, then the symmetric capacity is $\frac{\Delta}{2 \Delta+1}$, where $\Delta$ is the minimum internal conflict distance.

Proof: The case where each alignment set contains no overlapping cycles, i.e., no edge participates in more than one cycle, is dealt with more generally (no constraints on the number of edges) in Section II-C in Theorem 8 . Here we only consider the remaining cases where the alignment graph has overlapping cycles, as shown in Fig. 4. We rely on a linear scheme over $2 \Delta+1$ channel uses and send $\Delta$ symbols for each message. When $\Delta=1, \frac{\Delta}{2 \Delta+1}=\frac{1}{3}$ can be achieved easily by multicast (CDMA) as each receiver is interfered by at most two messages. This is because over three channel uses, each receiver sees three generic linear equations in the three symbols (one desired, two interfering) that it is 


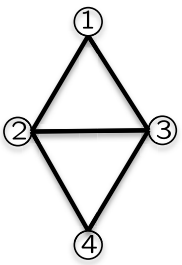

(a)

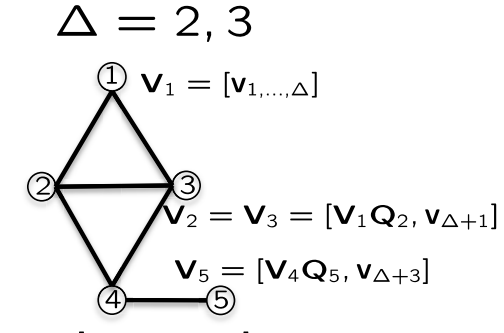

$\mathbf{V}_{4}=\left[\mathbf{V}_{2} \mathbf{Q}_{4}, \mathbf{v}_{\Delta+2}\right]$

(e)

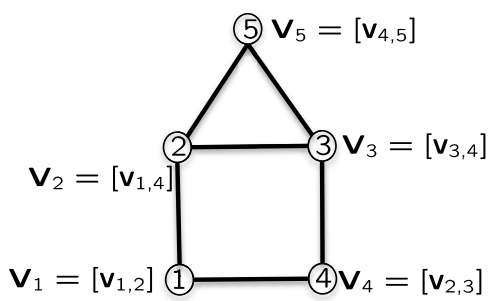

(c)

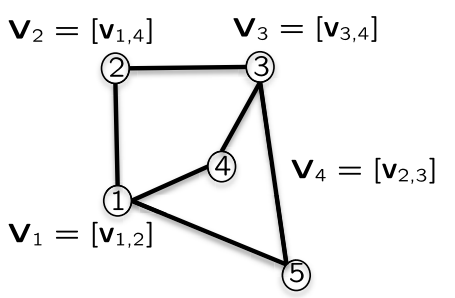

(d)
$\mathbf{V}_{5}=\left[\mathbf{V}_{1} \mathbf{Q}_{5}, \mathbf{V}_{3} \mathbf{Q}_{6}\right]$

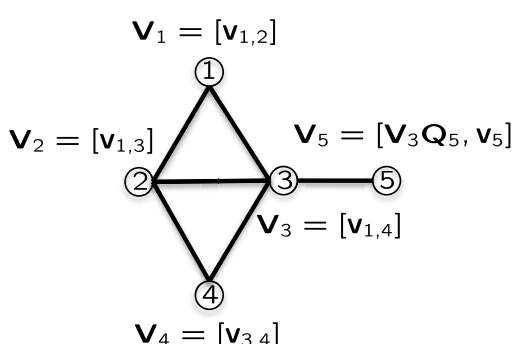

(f)

Fig. 4. All alignment graphs with 6 or fewer edges and containing overlapping cycles. Also shown is a vector space assignment solution that avoids all conflicts at distance $\Delta$ or more.

able to hear, from which it can resolve all three. Now consider $\Delta \geq 2$. The achievable scheme for each alignment set is shown in Fig. 4. Note that case (a) is a subcase of case (e), allowing the same solution, and case (b) cannot have conflict distance more than 1 . For all remaining cases, only (e) can have conflict distance 3 . The $\mathbf{v}_{i}$ are $(2 \Delta+1) \times 1$ column vectors that are in general position, and all $\mathbf{Q}_{i}$ are generic transformations with dimension $\Delta \times(\Delta-1)$. It is easily verified that each alignment edge occupies no more than $\Delta+1$ dimensions and all internal conflicts are avoided.

Note that for case (f), we assume that if $W_{5}$ is interfered by two messages within the alignment set, the interfering messages are $W_{1}, W_{2}$ instead of $W_{2}, W_{4}$, without loss of generality. As this is a multiple unicast setting, it is not possible for $W_{1}, W_{2}$ and $W_{2}, W_{4}$ to be interference at $W_{5}$ simultaneously. But if we go to multiple groupcast setting, this is possible and we can construct an instance that exhibits similar properties as the instance shown in Fig. 1. Specifically, we will present a simplest example where non-Shannon inequalities are needed for multiple groupcast index coding problems in the next section.

5) Multiple Groupcast - The Simplest Example: As mentioned previously, Blasiak et al. have presented the first example (and the only example previously identified) of a multiple groupcast index coding problem in [8] where non-Shannon inequalities are necessary. The example presented by Blasiak et al. is based directly on the Vamos matroid, so that it contains 8 messages and 200 receivers (one for every element of every circuit). In this section we use the interference alignment perspective to identify the simplest such example for multiple groupcast index coding. Our new criteria for the simplest example are the following.

Criteria for the Simplest Example:

1) Requires non-Shannon inequalities.

2) Each receiver sees no more than 2 interferers (one-to-one alignment demands).
3) Has the minimum number of edges in its alignment graph (among all examples that satisfy the first two criteria).

Note that the restriction to multiple unicast is removed.

As we will show, the simplest example, shown in Fig. 5, has only 6 messages (as opposed to 8 messages in the groupcast example of [8] and 11 messages in the simplest unicast example), only 6 alignment edges in the alignment graph (as opposed to 7 in the simplest unicast example), and a total of only 10 receivers (as opposed to 200 receivers in the groupcast example of [8] and 11 receivers in the simplest unicast example). As the core part (the diamond $W_{2}, W_{3}, W_{4}, W_{5}$ ) for the necessity of non-Shannon inequalities remains the same, the intuition and proofs follow previous discussions. We establish the necessity of non-Shannon inequalities and find the linear capacity with the following theorem.

Theorem 6: For the index coding instance shown in Fig. 5, the best possible outer bound value on the symmetric capacity from only Shannon inequalities is $\frac{2}{5}$, whereas the Zhang-Yeung non-Shannon-type information inequality yields the tighter outer bound value of $\frac{11}{28}$. Moreover, the symmetric linear capacity is $\frac{5}{13}$.

The proof of Theorem 6 follows along the same lines as the proofs for Theorem 3 and Theorem 4. Details are relegated to Section IV-E. The optimal linear achievability scheme, shown in Fig. 6, has an interesting aspect that even though the alignment demands are one-to-one, the optimal solution requires subspace alignments. Evidently, subspace alignment solutions may be required even when all demands are only one-to-one alignment demands.

Next we prove that this is the simplest example for multiplegroupcast index coding problems. This is shown by the observation that all simpler cases are already solved without the need for non-Shannon inequalities. For half-rate-feasible networks, the capacity is already known and only Shannon inequalities are required [3]. For half-rate-infeasible networks, 


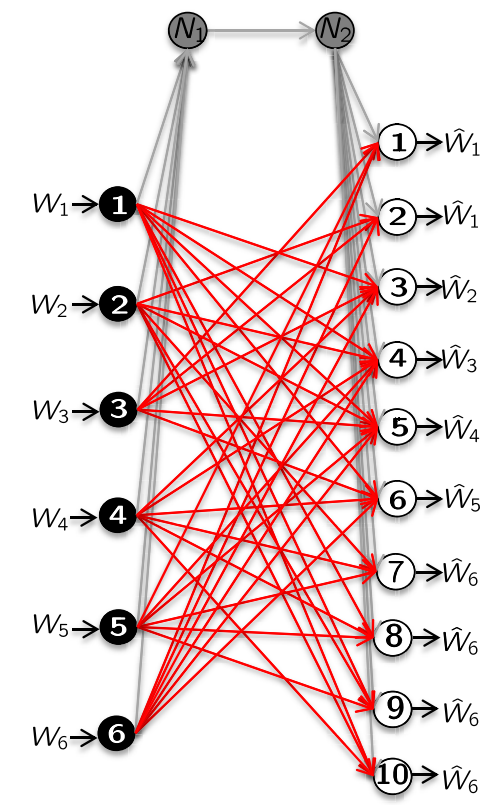

(a)

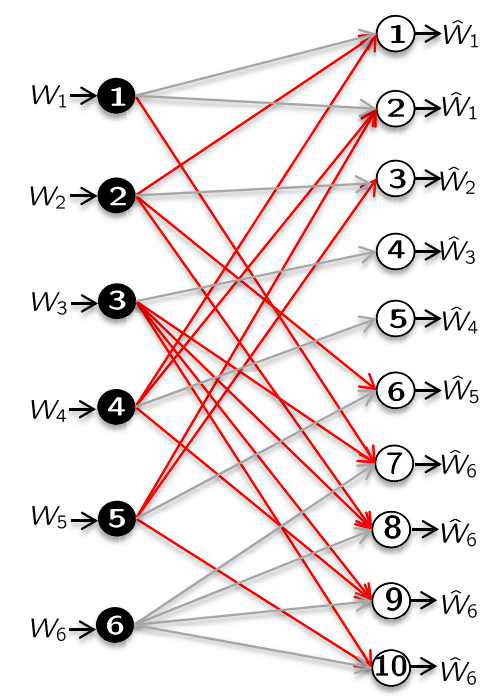

(b)
Interference at

each destination

$\left\{W_{2}, W_{4}\right\}$

$\left\{W_{4}, W_{5}\right\}$

$\left\{W_{5}\right\}$

$\{\emptyset\}$

$\{\emptyset\}$

$\left\{W_{2}\right\}$

$\left\{W_{1}, W_{3}\right\}$

$\left\{W_{2}, W_{3}\right\}$

$\left\{W_{3}, W_{4}\right\}$

$\left\{W_{3}, W_{5}\right\}$

(c)

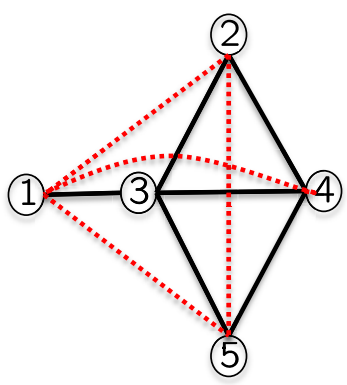

(6)

(d)

Fig. 5. The Simplest Example of a Multiple Groupcast Index Coding Problem where Non-Shannon Inequalities are Necessary. (a) Antidote Graph. (b) Interference Graph. (c) List of interferers at each destination. (d) Alignment and Conflict Graphs.

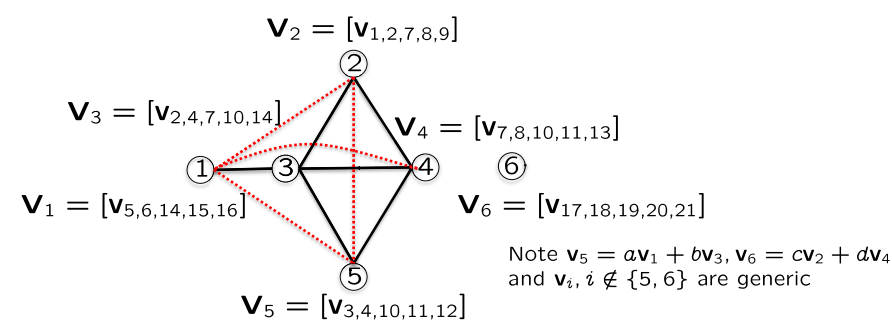

Fig. 6. A vector space assignment that avoids all conflicts. Symmetric rate achieved is $5 / 13$ per message. $\mathbf{v}_{i}, \mathbf{v}_{j}$ is abbreviated as $\mathbf{v}_{i, j}$, etc. $\mathbf{v}_{i}, i \neq 5,6$ are generic $13 \times 1$ vectors and $a, b, c, d$ are generic scalars.

if the number of interferers seen by each destination is not more than 2, and there are fewer than 6 alignment edges in an alignment set, then the following theorem shows that the conflict bound (also based only on Shannon inequalities) is tight.

Theorem 7: For the class of half-rate-infeasible multiplegroupcast index coding problems where each destination is interfered by at most two messages, if each alignment set contains fewer than 6 alignment edges, then the symmetric capacity is $\frac{\Delta}{2 \Delta+1}$, where $\Delta$ is the minimum internal conflict distance.

Proof: Among such cases, case (a) in Fig. 4 is the only one with overlapping cycles. The achievable scheme follows from case (e) with $\Delta=2$ in Fig. 4. Cases with no overlapping cycles are dealt with separately in Theorem 8 .

Remark: It is not difficult to verify that the multiple groupcast index coding network of Fig. 5 is also the simplest network if instead of the minimum number of alignment edges, we require the minimum number of messages.

\section{Expanding the Interference Alignment Perspective}

Recall that [3] presents a characterization of the multiple groupcast index coding capacity for all instances where

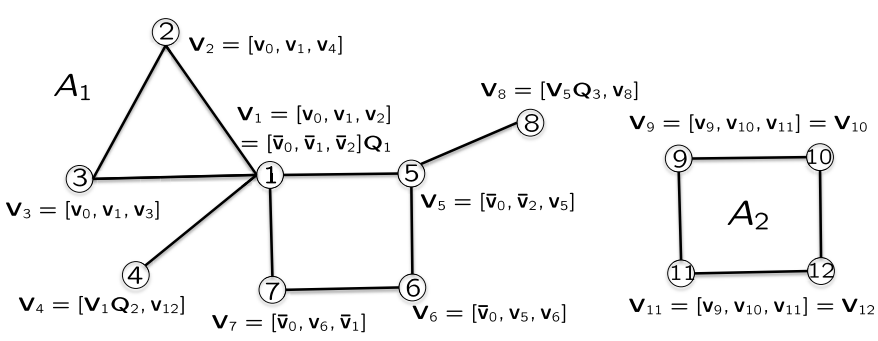

Fig. 7. An alignment graph showing a solution that avoids all conflicts at distance 3 . Symmetric rate achieved is $3 / 7$ per message.

each connected component of the alignment graph, i.e., each alignment set, either does not contain a cycle or does not contain a fork. The next step to expand this class is to also allow alignment sets to contain both cycles and forks. In our search for the simplest example, we were able to find such an extension. The result is presented as the next theorem.

Theorem 8: For the class of half-rate-infeasible multiplegroupcast index coding problems where each destination is interfered by at most two messages, if each alignment set has no overlapping cycles, i.e., no two cycles share an edge, then the symmetric capacity is $\frac{\Delta}{2 \Delta+1}$, where $\Delta$ is the minimum internal conflict distance.

In [3], forks are handled by inheriting vectors from parent nodes, and cycles are handled by cyclic assignments of vector spaces. Here, we combine both techniques to simultaneously deal with cycles and forks.

As an illustration of the result, an alignment graph is shown in Fig. 7. While conflicts are not shown, the minimum internal conflict distance is assumed to be 3 . Note that the assignment of precoding vectors avoids all conflicts at distance 3 or more. Two alignment sets are shown, labeled as $A_{1}, A_{2}$. $A_{2}$ cannot have an internal conflict because the minimum 


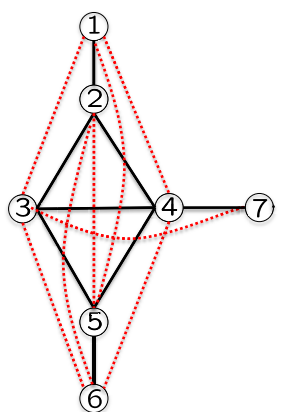

(a)
Interference At Each Destination

$(8)$
$(9)$
$(10)$
(11)
(12)

$$
\begin{array}{ll}
1:\left\{W_{3}, W_{4}\right\} & 8:\left\{W_{2}, W_{3}\right\} \\
2:\left\{W_{5}, W_{6}\right\} & 9:\left\{W_{2}, W_{4}\right\} \\
3:\{\emptyset\} & 10:\left\{W_{3}, W_{5}\right\} \\
4:\{\emptyset\} & 11:\left\{W_{4}, W_{5}\right\} \\
5:\left\{W_{1}, W_{2}\right\} & 12:\left\{W_{4}, W_{7}\right\} \\
6:\left\{W_{3}, W_{4}\right\} & \\
7:\left\{W_{3}\right\} &
\end{array}
$$

Fig. 8. A multiple unicast index coding problem with 8 edges in the alignment graph where non-Shannon inequalities are necessary. (a) Alignment and conflict graphs, and (b) Only the list of interferers at each destination is shown for simplicity.

conflict distance is 3 . All $\mathbf{v}_{i}$ are $7 \times 1$ vectors in general position. $\mathbf{Q}_{1}$ is a generic $3 \times 3$ matrix and $\mathbf{Q}_{2}, \mathbf{Q}_{3}$ are generic $3 \times 2$ matrices, meant to extract a generic 2 dimensional subspace from the connected node's signal space. Each message occupies $3 / 7$ dimensions and any two adjacent messages occupy no more than $4 / 7$ dimensions, leaving the remaining $3 / 7$ dimensions for the desired signal. Note that for cycles, some randomly permuted common vectors are used combined with cyclicly assigned vectors. Detailed proof is presented in Section IV-F.

\section{DISCUSSION}

While in this work we focused on the simplest instances, the necessity of non-Shannon inequalities can appear in different settings beyond the simplest examples. For example, Fig. 8 shows an interesting example with 8 alignment edges in the alignment graph. This is a multiple unicast index coding problem with 12 messages. While the inner diamond remains the same as previous examples, the supporting edges appear in different positions relative to the diamond. Using the same approach, we get corresponding results for this network - although the best outer bound based on only Shannon inequalities is $\frac{2}{5}$, we can tighten it in this case to $\frac{13}{33}$ with the Zhang-Yeung non-Shannon type information inequality and the linear capacity, in this case, is $\frac{7}{18}$, which is shown using the Ingleton inequality. The proof follows along similar lines of the proofs already presented.

An interesting direction for future work would be to go beyond symmetric rates. For example, one could try to find the simplest example where non-Shannon information inequalities are needed to settle the entire capacity region. While we expect that the techniques presented in this work will be useful for such extensions, it is likely that additional insights may be involved.

While non-Shannon inequalities have been extensively studied, they are still not well understood both with regards to their fundamental character and their practical implications. The natural perspective provided by interference alignment can be quite valuable in both regards. It is tempting to interpret the non-Shannon inequalities, from an interference alignment perspective, as representing precisely the possibilities of subspace alignments. As the number of messages grows, so does the number of possibilities in how these messages can align with each other. Much like the curious resemblance between the alignment graph of our example which is motivated by the interference alignment perspective, and the Vamos matroid, there are noticeable parallels between the observations made through interference alignment and the fundamental information inequalities. As another example, consider first the observation that while there are only finitely many information inequalities for up to 3 random variables, the number of information inequalities grows to infinity when 4 or more variables are involved. This observation could be related to the existence of non-asymptotic interference alignment solutions for generic channels in the $K$ user interference channel for up to 3 users, but not beyond 3 users.

It is notable that in the degrees of freedom studies of wireless networks with generic channels, sufficient diversity, and full channel knowledge, the need for non-Shannon inequalities has not yet surfaced. The essential connection between the topological interference management problem and the index coding problem that is highlighted in [3], does link the results of this work to wireless networks with channel uncertainty. However, it is still not clear that non-Shannon inequalities are necessary for the topological interference management problem. This is because, unlike the index coding problem where all messages can be encoded together, there is an additional constraint that the encoding of messages must be done in a distributed fashion in an interference network. It is possible that the additional constraint may manifest itself purely through submodularity to produce tighter outer bounds. The distributed coding requirement combined with the linear channel also favors the optimality of linear solutions in interference networks. Whether the linear coding capacity found for our simplest examples is the true capacity of these instance of the index coding problem, or for the corresponding instances of the topological interference management problem, remains a mystery.

\section{PROOFS}

\section{A. Derivation of $(*)$}

Suppose $A, B$ are vector spaces. It is well known that

$$
\operatorname{dim}(A \cap B)=\operatorname{dim}(A)+\operatorname{dim}(B)-\operatorname{dim}(A \cup B)
$$

Restricting to only the subspace projections within vector space $C$, it follows that

$$
\begin{aligned}
\operatorname{dim}((A \cap B) \cap C)= & \operatorname{dim}(A \cap C)+\operatorname{dim}(B \cap C) \\
& -\operatorname{dim}((A \cup B) \cap C)
\end{aligned}
$$

We can bound the LHS and RHS of (4) as follows.

$$
\begin{aligned}
& L H S \leq \operatorname{dim}(A \cap B) \\
& R H S \geq \operatorname{dim}(A \cap C)+\operatorname{dim}(B \cap C)-\operatorname{dim}(C)
\end{aligned}
$$

which gives us

$$
\operatorname{dim}(A \cap B) \geq \operatorname{dim}(A \cap C)+\operatorname{dim}(B \cap C)-\operatorname{dim}(C)
$$

Substituting $A \triangleq \mathbf{V}_{2}, B \triangleq \mathbf{V}_{5}, C \triangleq \mathbf{V}_{3} \cap \mathbf{V}_{4}$, we have the desired statement. 


\section{B. Proof for Theorem 2}

Proof $\left(C_{o} \leq \frac{2}{5}\right)$ : From the alignment graph shown in Fig. 1(d), the minimum internal conflict distance $\Delta=2$. From [3, Th. 6], we therefore have the symmetric capacity outer bound $\frac{\Delta}{2 \Delta+1}=\frac{2}{5}$. Since the internal-conflict bound is obtained only from Shannon-type inequalities, the best Shannon-type outer bound $C_{o} \leq \frac{2}{5}$.

Proof $\left(C_{o} \geq \frac{2}{5}\right)$ : For this proof, we will construct a sub-modular $T \in \mathcal{T}$ which corresponds to the symmetric rate $\frac{2}{5}$, thus proving that $\left(R_{1}, R_{2}, \cdots, R_{N}\right)=\left(\frac{2}{5}, \frac{2}{5}, \cdots, \frac{2}{5}\right)$ is contained in $\mathcal{C}_{o}$. This in turn implies that $C_{o} \geq \frac{2}{5}$.

The $T$ function is constructed as follows.

$$
\begin{aligned}
T_{\varnothing} & =0, \quad T_{i}=\frac{2}{5}, \quad \forall i \in\{1,2, \ldots, 11\} \\
T_{1,3} & =T_{2,3}=T_{2,4}=T_{3,4}=T_{3,5}=T_{4,5}=T_{4,6}=\frac{3}{5}, \\
T_{i, j} & =\frac{4}{5} \text { for all the other } i, j \\
T_{1,2,3} & =T_{1,3,5}=T_{1,3,4}=T_{2,3,4}=T_{3,4,5}=T_{2,3,5} \\
& =T_{2,4,5}=T_{3,4,6}=T_{2,4,6}=T_{4,5,6}=\frac{4}{5} \\
T_{i, j, k} & =1 \text { for all the other } i, j, k \\
T_{2,3,4,5} & =\frac{4}{5} \text { and the } T \text { function is } 1 \text { everywhere else. }
\end{aligned}
$$

For the index coding instance of Fig. 1, it is trivial to check $R_{j}=\frac{2}{5} \leq T_{\{j\} \cup \mathcal{B}_{j}}-T_{\mathcal{B}_{j}}$. Therefore, all the remains to be shown is that $T \in \mathcal{T}$. It is easy to see that $T_{\varnothing}=0, T_{\{1,2, \ldots, 11\}}=1$ and $T_{\mathcal{J}} \leq T_{\mathcal{K}}, \forall \mathcal{J} \subset \mathcal{K}$. We are only left to show the submodularity property, i.e., $T_{\mathcal{J}}+T_{\mathcal{K}} \geq T_{\mathcal{J} \cup \mathcal{K}}+T_{\mathcal{J} \cap \mathcal{K}}, \forall \mathcal{J}, \mathcal{K}$. Without loss of generality, we assume $|\mathcal{J}| \leq|\mathcal{K}|$. We note that submodularity holds for two special cases trivially, i.e., $\mathcal{J} \cap \mathcal{K}=\emptyset$ or $\mathcal{J} \subset \mathcal{K}$. Henceforth we consider only the cases where $\mathcal{J}$ intersects with, but does not belong to $\mathcal{K}$, i.e., $|\mathcal{J}| \geq 2$.

If $|\mathcal{J}|=|\mathcal{K}|=2, T_{\mathcal{J}}+T_{\mathcal{K}} \geq T_{\mathcal{J} \cup \mathcal{K}}+T_{\mathcal{J} \cap \mathcal{K}}$ holds when $T_{\mathcal{J}}=T_{\mathcal{K}}=\frac{3}{5}$ as $T_{\mathcal{J} \cup \mathcal{K}}=\frac{4}{5}$ from (10) and $T_{\mathcal{J} \cap \mathcal{K}}=T_{i}=\frac{2}{5}$. And also all the other cases as $T_{\mathcal{J}}+T_{\mathcal{K}} \geq \frac{3}{5}+\frac{4}{5} \geq 1+\frac{2}{5} \geq$ $T_{\mathcal{J} \cup \mathcal{K}}+T_{\mathcal{J} \cap \mathcal{K}}$.

If $|\mathcal{J}|=2,|\mathcal{K}|=3$, submodularity also holds as $|\mathcal{J} \cap \mathcal{K}|=1, T_{\mathcal{J} \cap \mathcal{K}}=\frac{2}{5}$ and $T_{\mathcal{J}}+T_{\mathcal{K}} \geq \frac{3}{5}+\frac{4}{5} \geq 1+\frac{2}{5} \geq$ $T_{\mathcal{J} \cup \mathcal{K}}+T_{\mathcal{J} \cap \mathcal{K}}$. Similar reason applies when $|\mathcal{J}|=2,|\mathcal{K}| \geq 4$.

If $|\mathcal{J}|=|\mathcal{K}|=3$, submodularity holds whether $|\mathcal{J} \cap \mathcal{K}|=1$ (trivial) or $|\mathcal{J} \cap \mathcal{K}|=2$ as $T_{\mathcal{J} \cap \mathcal{K}}=\frac{3}{5}$ from (9) with the only exception $T_{2,3,5}+T_{2,4,5}=\frac{8}{5} \geq T_{2,3,4,5}+T_{2,5}=\frac{8}{5}$.

Consider $|\mathcal{J}|=3, \mathcal{K}=\{2,3,4,5\}$. If further $T_{\mathcal{J}}=\frac{4}{5}$, all possible intersections belong to (9) and $T_{\mathcal{J} \cap \mathcal{K}}=\frac{3}{5}$, satisfying submodularity. Otherwise $T_{\mathcal{J}}=1, T_{\mathcal{J}}+T_{\mathcal{K}} \geq 1+\frac{4}{5} \geq$ $T_{\mathcal{J} \cup \mathcal{K}}+T_{\mathcal{J} \cap \mathcal{K}}$. This formula also holds for all the other cases where $|\mathcal{K}| \geq 4$.

For all cases that remain, $T_{\mathcal{J}}=T_{\mathcal{K}}=1$ and submodularity follows trivially. This completes the proof for polymadroid upper bound.

\section{Proof for Theorem 3}

Now we proceed to the tighter outer bound where we will use non-Shannon information inequalities. The proof involves two parts, only the second part will involve a non-Shannon inequality. We start with the first part. Each solid black edge in the alignment graph represents an interference union at a desired destination. In order to leave $R$ dimensions for the desired message, the interference union must be compressed to a space with dimension smaller than $1-R$. This is made information theoretically rigorous as follows. Consider an alignment edge $(i, j)$ in the alignment graph and their interfering destination $k$ that desires $W_{k}$. As destination $k$ has all the messages except $W_{i}, W_{j}, W_{k}$ (denoted by $W_{i, j, k}^{c}$ ) as antidotes, from Fano's inequality, we have

$$
\begin{aligned}
& n R_{k} \leq I\left(W_{k} ; \mathcal{S}^{n}, W_{i, j, k}^{c}\right)+o(n) \\
& \leq I\left(W_{k} ; \mathcal{S}^{n} \mid W_{i, j, k}^{c}\right)+o(n) \\
& \leq H\left(\mathcal{S}^{n} \mid W_{i, j, k}^{c}\right)-H\left(\mathcal{S}^{n} \mid W_{i, j}^{c}\right)+o(n) \\
& \leq n-H\left(\mathcal{S}^{n} \mid W_{i, j}^{c}\right)+o(n) \\
\Rightarrow & H\left(\mathcal{S}^{n} \mid W_{i, j}^{c}\right) \leq n\left(1-R_{k}\right)+o(n)
\end{aligned}
$$

where (14) follows from the independence between the messages and (16) is due to the fact that $\mathcal{S}$ carries one symbol per channel use. We also get a byproduct from (15),

$$
H\left(\mathcal{S}^{n} \mid W_{i, j, k}^{c}\right) \geq H\left(\mathcal{S}^{n} \mid W_{i, j}^{c}\right)+n R_{k}+o(n)
$$

whose meaning is that the interference space $H\left(\mathcal{S}^{n} \mid W_{i, j}^{c}\right)$ is separable from the desired signal space.

Giving all the other messages except the desired $W_{k}$ as antidotes to destination $k$, we have

$$
\begin{aligned}
n R_{k} & \leq I\left(W_{k} ; \mathcal{S}^{n} \mid W_{k}^{c}\right)+o(n) \\
& \leq H\left(\mathcal{S}^{n} \mid W_{k}^{c}\right)-H\left(\mathcal{S}^{n} \mid W_{\emptyset}^{c}\right)+o(n) \\
& \leq H\left(\mathcal{S}^{n} \mid W_{k}^{c}\right)+o(n)
\end{aligned}
$$

where (21) follows from the observation that $\mathcal{S}^{n}$ is a function of all the messages and this intuitively states that the information contained in $\mathcal{S}^{n}$ cannot be smaller than the entropy of desired message $W_{k}$ when the uncertainty due to the other messages is not present.

For the alignment chain $W_{1}-W_{3}-W_{4,5}$, we have

$$
\begin{aligned}
& H\left(\mathcal{S}^{n} \mid W_{1,3}^{c}\right)+H\left(\mathcal{S}^{n} \mid W_{3,4,5}^{c}\right) \\
& \quad \geq H\left(\mathcal{S}^{n} \mid W_{3}^{c}\right)+H\left(\mathcal{S}^{n} \mid W_{1,3,4,5}^{c}\right) \\
& \quad \geq H\left(\mathcal{S}^{n} \mid W_{3}^{c}\right)+H\left(\mathcal{S}^{n} \mid W_{1,4,5}^{c}\right) \\
& \quad \geq H\left(\mathcal{S}^{n} \mid W_{3}^{c}\right)+n R_{1}+H\left(\mathcal{S}^{n} \mid W_{4,5}^{c}\right)+o(n) \\
& \quad \geq n R_{3}+n R_{1}+H\left(\mathcal{S}^{n} \mid W_{4,5}^{c}\right)+o(n) \\
& \Rightarrow H\left(\mathcal{S}^{n} \mid W_{3,4,5}^{c}\right) \geq n(3 R-1)+H\left(\mathcal{S}^{n} \mid W_{4,5}^{c}\right)+o(n)
\end{aligned}
$$

where (22) follows from submodularity, (23) is due to the fact that conditioning reduces entropy, (24) follows from (18) with $i=4, j=5, k=1$, (25) follows from (21) with $k=3$ and (26) is due to (17) with $i=1, j=3$ and because we are only interested in symmetric capacity.

Symmetrically, for the alignment chain $W_{6}-W_{4}-W_{2,3}$, we have

$$
H\left(\mathcal{S}^{n} \mid W_{2,3,4}^{c}\right) \geq n(3 R-1)+H\left(\mathcal{S}^{n} \mid W_{2,3}^{c}\right)+o(n)
$$




$$
\begin{aligned}
& 3 H\left(\mathcal{S}^{n}, W_{2,4}^{c}\right)+3 H\left(\mathcal{S}^{n}, W_{4,5}^{c}\right)+3 H\left(\mathcal{S}^{n}, W_{3,4}^{c}\right)+H\left(\mathcal{S}^{n}, W_{2,3}^{c}\right)+H\left(\mathcal{S}^{n}, W_{3,5}^{c}\right) \\
& \geq 2 H\left(\mathcal{S}^{n}, W_{2,3,4}^{c}\right)+2 H\left(\mathcal{S}^{n}, W_{3,4,5}^{c}\right)+H\left(\mathcal{S}^{n}, W_{2,5}^{c}\right)+H\left(\mathcal{S}^{n}, W_{2,4,5}^{c}\right)+H\left(\mathcal{S}^{n}, W_{3}^{c}\right)+4 H\left(\mathcal{S}^{n}, W_{4}^{c}\right) \\
\Rightarrow & 3 H\left(\mathcal{S}^{n} \mid W_{2,4}^{c}\right)+3 H\left(\mathcal{S}^{n} \mid W_{4,5}^{c}\right)+3 H\left(\mathcal{S}^{n} \mid W_{3,4}^{c}\right)+H\left(\mathcal{S}^{n} \mid W_{2,3}^{c}\right)+H\left(\mathcal{S}^{n} \mid W_{3,5}^{c}\right) \\
& \geq 2 H\left(\mathcal{S}^{n} \mid W_{2,3,4}^{c}\right)+2 H\left(\mathcal{S}^{n} \mid W_{3,4,5}^{c}\right)+H\left(\mathcal{S}^{n} \mid W_{2,5}^{c}\right)+H\left(\mathcal{S}^{n} \mid W_{2,4,5}^{c}\right)+H\left(\mathcal{S}^{n} \mid W_{3}^{c}\right)+4 H\left(\mathcal{S}^{n} \mid W_{4}^{c}\right) \\
\Rightarrow & 7 n(1-R)+3 H\left(\mathcal{S}^{n} \mid W_{4,5}^{c}\right)+H\left(\mathcal{S}^{n} \mid W_{2,3}^{c}\right) \geq 2 H\left(\mathcal{S}^{n} \mid W_{2,3,4}^{c}\right)+2 H\left(\mathcal{S}^{n} \mid W_{3,4,5}^{c}\right)+2 H\left(\mathcal{S}^{n} \mid W_{2,5}^{c}\right)+5 n R+o(n) \\
\Rightarrow & 7 n(1-R)+3 H\left(\mathcal{S}^{n} \mid W_{4,5}^{c}\right)+H\left(\mathcal{S}^{n} \mid W_{2,3}^{c}\right) \geq 2 H\left(\mathcal{S}^{n} \mid W_{2,3,4}^{c}\right)+2 H\left(\mathcal{S}^{n} \mid W_{3,4,5}^{c}\right)+9 n R+o(n) \\
\Rightarrow & n(7-16 R)+3 H\left(\mathcal{S}^{n} \mid W_{4,5}^{c}\right)+H\left(\mathcal{S}^{n} \mid W_{2,3}^{c}\right) \geq 2 H\left(\mathcal{S}^{n} \mid W_{2,3,4}^{c}\right)+2 H\left(\mathcal{S}^{n} \mid W_{3,4,5}^{c}\right)+o(n)
\end{aligned}
$$

and adding (26) and (27), we arrive at the final formula for the first part

$$
\begin{aligned}
H\left(\mathcal{S}^{n} \mid W_{2,3,4}^{c}\right)+H\left(\mathcal{S}^{n} \mid W_{3,4,5}^{c}\right) \geq & n(6 R-2)+H\left(\mathcal{S}^{n} \mid W_{2,3}^{c}\right) \\
& +H\left(\mathcal{S}^{n} \mid W_{4,5}^{c}\right)+o(n) .
\end{aligned}
$$

We proceed to the deal with the diamond. As mentioned before, we need the Zhang-Yeung non-Shannon-type information inequality for 4 random variables, stated as follows.

Theorem 9: (Zhang-Yeung non-Shannon-type information inequality [18])

$$
\begin{aligned}
3 H & (A, C)+3 H(A, D)+3 H(C, D)+H(B, C)+H(B, D) \\
\geq & 2 H(C)+2 H(D)+H(A, B)+H(A) \\
& +H(B, C, D)+4 H(A, C, D)
\end{aligned}
$$

We assign the random variables as follows,

$$
\begin{array}{ll}
A=\mathcal{S}^{n}, W_{2,4,5}^{c}, & B=\mathcal{S}^{n}, W_{2,3,5}^{c}, \\
C=\mathcal{S}^{n}, W_{2,3,4}^{c}, & D=\mathcal{S}^{n}, W_{3,4,5}^{c} .
\end{array}
$$

Substituting into the Zhang-Yeung non-Shannon-type information inequality, we have (31) shown at the top of this page, where (32) follows from the independence of the messages and that the number of messages is the same on the LHS and the RHS, the LHS of (33) follows from the fact that $(2,4),(3,4),(3,5)$ are all alignment edges and use (17), the RHS of (33) follows from the principle that conditioning reduces entropy and uses (21) with $k=3,4$ and (34) follows from (18) with $i=2, j=\emptyset, k=5$ and from (21) with $k=2$.

Next, let us switch the value of $A, B$, i.e., $A=\mathcal{S}^{n}, W_{2,3,5}^{c}, B=\mathcal{S}^{n}, W_{2,4,5}^{c}, C=\mathcal{S}^{n}, W_{2,3,4}^{c}$, $D=\mathcal{S}^{n}, W_{3,4,5}^{c}$ and similar to (35), we have

$$
\begin{aligned}
n(7-16 R) & +3 H\left(\mathcal{S}^{n} \mid W_{2,3}^{c}\right)+H\left(\mathcal{S}^{n} \mid W_{4,5}^{c}\right) \\
\geq & 2 H\left(\mathcal{S}^{n} \mid W_{2,3,4}^{c}\right)+2 H\left(\mathcal{S}^{n} \mid W_{3,4,5}^{c}\right)+o(n) .
\end{aligned}
$$

Adding (35) and (36) and dividing by 2 on both sides, we have the final formula for the second part,

$$
\begin{aligned}
& n(7-16 R)+2 H\left(\mathcal{S}^{n} \mid W_{2,3}^{c}\right)+2 H\left(\mathcal{S}^{n} \mid W_{4,5}^{c}\right) \\
& \geq 2 H\left(\mathcal{S}^{n} \mid W_{2,3,4}^{c}\right)+2 H\left(\mathcal{S}^{n} \mid W_{3,4,5}^{c}\right)+o(n) .
\end{aligned}
$$

Adding (37) with 2 times (28) and normalizing by $n$, we arrive at the conclusion

$$
7-16 R \geq 2(6 R-2) \Rightarrow R \leq \frac{11}{28}
$$

which completes the proof.

\section{Proof for Theorem 4}

The proof of the linear outer bound follows along similar lines as the information theoretical outer bound, and also consists of two parts. As linear capacity must be less than the actual capacity, the outer bound for the information theoretical outer bound can be directly applied here. We directly borrow the conclusion of the first part as follows according to (28),

$$
\begin{aligned}
\operatorname{dim}\left(\mathbf{V}_{2,3,4}\right)+\operatorname{dim}\left(\mathbf{V}_{3,4,5}\right) \geq & 6 R-2+\operatorname{dim}\left(\mathbf{V}_{2,3}\right) \\
& +\operatorname{dim}\left(\mathbf{V}_{4,5}\right) .
\end{aligned}
$$

Note that the entropy space term $H\left(\mathcal{S}^{n} \mid W_{i, j, k}^{c}\right)$ is replaced by the vector space form $\mathbf{V}_{i, j, k}$.

For the second part, i.e., the diamond, we resort to the Ingleton inequality for a tighter linear bound. Note that there exist some joint distributions of four random variables that violate the Ingleton inequality [17], so the Ingleton bound does not hold information theoretically. However, it does hold for all vector spaces, so we can use it for this proof. From the Ingleton inequality (3), we have

$$
\begin{gathered}
\operatorname{dim}\left(\mathbf{V}_{2,3}\right)+\operatorname{dim}\left(\mathbf{V}_{2,4}\right)+\operatorname{dim}\left(\mathbf{V}_{4,5}\right)+\operatorname{dim}\left(\mathbf{V}_{3,5}\right)+\operatorname{dim}\left(\mathbf{V}_{3,4}\right) \\
\geq \operatorname{dim}\left(\mathbf{V}_{3}\right)+\operatorname{dim}\left(\mathbf{V}_{4}\right)+\operatorname{dim}\left(\mathbf{V}_{2,5}\right) \\
\quad+\operatorname{dim}\left(\mathbf{V}_{2,3,4}\right)+\operatorname{dim}\left(\mathbf{V}_{3,4,5}\right) \\
\Rightarrow \operatorname{dim}\left(\mathbf{V}_{2,3}\right)+\operatorname{dim}\left(\mathbf{V}_{4,5}\right)+3(1-R) \geq 2 R+\operatorname{dim}\left(\mathbf{V}_{2}\right) \\
\quad+\operatorname{dim}\left(\mathbf{V}_{5}\right)+\operatorname{dim}\left(\mathbf{V}_{2,3,4}\right)+\operatorname{dim}\left(\mathbf{V}_{3,4,5}\right) \\
\Rightarrow \operatorname{dim}\left(\mathbf{V}_{2,3}\right)+\operatorname{dim}\left(\mathbf{V}_{4,5}\right)+3-7 R \\
\geq \operatorname{dim}\left(\mathbf{V}_{2,3,4}\right)+\operatorname{dim}\left(\mathbf{V}_{3,4,5}\right)
\end{gathered}
$$

where (41) follows from (17) where $(2,4),(3,4),(3,5)$ are all interference edges, (21) with $k=3,4$ and there is conflict between $W_{2}$ and $W_{5},(42)$ is due to (21) with $k=2,5$.

Adding (39) and (42), we have

$$
3-7 R \geq 6 R-2 \Rightarrow R \leq \frac{5}{13}
$$

which completes the proof.

\section{E. Proof for Theorem 6}

The polymatroid upper bound $\frac{2}{5}$ is proved by explicitly assigning the spaces that correspond to each message and their unions with proper dimensions. Note that we need a groupcast version of $T$ function [14], which can be constructed similarly and follows the same rules as defined in [14]. The $T$ function 
is defined over the power set $\{1,2,3,4,5,6\}$ and we assign its values as follows. It satisfies the same constraints as specified in a previous proof in Section IV-B, i.e., $T \in \mathcal{T}$.

$$
\begin{aligned}
T_{\emptyset} & =0, \quad T_{i}=\frac{2}{5}, \quad \forall i \in\{1,2,3,4,5,6\} \\
T_{1,3} & =T_{2,3}=T_{2,4}=T_{3,4}=T_{3,5}=T_{4,5}=\frac{3}{5} \\
T_{i, j} & =\frac{4}{5} \text { for all the other } i, j \\
T_{1,2,3} & =T_{1,3,5}=T_{1,3,4}=T_{2,3,4}=T_{3,4,5}=T_{2,3,5} \\
& =T_{2,4,5}=\frac{4}{5}, \quad T_{i, j, k}=1 \text { for all the other } i, j, k
\end{aligned}
$$

$$
\begin{aligned}
& T_{2,3,4,5}=\frac{4}{5} \text { and all remaining unspecified values } \\
& \text { of } T \text { function are set to } 1 \text {. }
\end{aligned}
$$

Next we proceed to the information theoretic outer bound. For alignment chain $W_{1}-W_{3}-W_{4,5}$, following similar steps as (26), we have

$$
H\left(\mathcal{S}^{n} \mid W_{3,4,5}^{c}\right) \geq n(3 R-1)+H\left(\mathcal{S}^{n} \mid W_{4,5}^{c}\right)+o(n) .
$$

Similarly, for alignment chain $W_{1}-W_{3}-W_{2,4}$,

$$
H\left(\mathcal{S}^{n} \mid W_{2,3,4}^{c}\right) \geq n(3 R-1)+H\left(\mathcal{S}^{n} \mid W_{2,4}^{c}\right)+o(n) .
$$

For the diamond, we use Zhang-Yeung non-Shannon inequality to get the following (refer to (35)),

$$
\begin{aligned}
2 H\left(\mathcal{S}^{n} \mid W_{2,4}^{c}\right)+2 H\left(\mathcal{S}^{n} \mid W_{4,5}^{c}\right)+n(7-16 R) \\
\geq 2 H\left(\mathcal{S}^{n} \mid W_{2,3,4}^{c}\right)+2 H\left(\mathcal{S}^{n} \mid W_{3,4,5}^{c}\right)+o(n) .
\end{aligned}
$$

Combining (48)(49)(50) and normalizing by $n$, we have

$$
7-16 R \geq 12 R-4 \Rightarrow R \leq \frac{11}{28} \text {. }
$$

Then we consider the linear capacity. With reference to (48) and (49), we have

$$
\begin{aligned}
\operatorname{dim}\left(\mathbf{V}_{2,3,4}\right)+\operatorname{dim}( & \left.\mathbf{V}_{3,4,5}\right) \\
& \geq 6 R-2+\operatorname{dim}\left(\mathbf{V}_{2,4}\right)+\operatorname{dim}\left(\mathbf{V}_{4,5}\right) .
\end{aligned}
$$

With Ingleton inequality (similar to (42)), we have

$$
\begin{aligned}
\operatorname{dim}\left(\mathbf{V}_{2,4}\right)+\operatorname{dim}\left(\mathbf{V}_{4,5}\right) & +3-7 R \\
& \geq \operatorname{dim}\left(\mathbf{V}_{2,3,4}\right)+\operatorname{dim}\left(\mathbf{V}_{3,4,5}\right) .
\end{aligned}
$$

Adding (52)(53), we arrive at

$$
3-7 R \geq 6 R-2 \Rightarrow R \leq \frac{5}{13} .
$$

\section{F. Proof for Theorem 8}

The outer bound is already available from [3, Th. 6]. We only prove the achievability of $\frac{\Delta}{2 \Delta+1}$.

The goal is to operate over $2 \Delta+1$ channel uses and choose $\Delta$ precoding vectors for each message, along which $\Delta$ symbols for that message will be sent. A key idea here is that the precoding for each alignment set is designed independently. So we will describe the precoding vector design for each type of alignment set. According to whether there are internal conflicts in each alignment set, we have two cases.
1) Alignment Sets With No Internal Conflicts: For each alignment set $A_{i}$ that has no internal conflicts, we randomly generate a $(2 \Delta+1) \times \Delta$ matrix $\mathbf{V}\left(A_{i}\right)$.

$$
\mathbf{V}\left(A_{i}\right)=\operatorname{rand}(2 \Delta+1, \Delta)
$$

where $\operatorname{rand}(a, b)$ is a function that returns a randomly generated $a \times b$ matrix. The same precoding matrix $\mathbf{V}\left(A_{i}\right)$ will be used by every message node in $A_{i}$. That is, $\Delta$ symbols for each message $W \in A_{i}$ will be sent along the $\Delta$ columns of $\mathbf{V}\left(A_{i}\right)$.

2) Alignment Sets With Internal Conflicts:

1) From each alignment set $A_{i}$, arbitrarily choose one message node, say, $W_{1}\left(A_{i}\right)$ to be the center node. For each center node $W_{1}\left(A_{i}\right)$, randomly and independently generate a $(2 \Delta+1) \times \Delta$ precoding matrix $\mathbf{V}_{1}\left(A_{i}\right)$ to be used by the node message.

$$
\mathbf{V}_{1}\left(A_{i}\right)=\operatorname{rand}(2 \Delta+1, \Delta)
$$

2) Consider all the cycles incident on $W_{1}\left(A_{i}\right)$ and name them as $C_{1}$ to $C_{K}$ where $K$ is the total number of cycles incident on node $W_{1}\left(A_{i}\right)$. Enqueue all the nodes in each cycle. Let the length of cycle $k$ be $\left|C_{k}\right|=l_{k}, k \in$ $\{1, \ldots, K\}$. Label the messages in cycle $k$ (in order) as $W_{1}\left(C_{k}\right), W_{2}\left(C_{k}\right), \cdots, W_{l_{k}}\left(C_{k}\right)$. Randomly permutate the precoding matrix $\mathbf{V}_{1}\left(A_{i}\right)$,

$$
\begin{aligned}
\mathbf{Q}_{k}\left(A_{i}\right)= & \operatorname{rand}(\Delta, \Delta) \\
\mathbf{V}_{1}^{k}\left(A_{i}\right)= & \mathbf{v}_{1}\left(A_{i}\right) \mathbf{Q}_{k}\left(A_{i}\right) \\
= & {\left[\mathbf{u}_{1}^{k}\left(C_{k}\right), \mathbf{u}_{2}^{k}\left(C_{k}\right), \ldots, \mathbf{u}_{\left(\Delta-\left\lfloor l_{k} / 2\right\rfloor\right)^{+}}^{k}\left(C_{k}\right),\right.} \\
& \left.\mathbf{v}_{1}^{k}\left(C_{k}\right), \mathbf{v}_{2}^{k}\left(C_{k}\right), \ldots, \mathbf{v}_{\Delta-\left(\Delta-\left\lfloor l_{k} / 2\right\rfloor\right)^{+}}^{k}\left(C_{k}\right)\right]
\end{aligned}
$$

Note that we divide the precoding vectors into two parts. The left $\left(\Delta-\left\lfloor l_{k} / 2\right\rfloor\right)^{+}$vectors would be the common vectors assigned to all the nodes in cycle $k$ and right $\Delta-\left(\Delta-\left\lfloor l_{k} / 2\right\rfloor\right)^{+}$vectors would be used in a cyclic fashion. If $\Delta \leq\left\lfloor l_{k} / 2\right\rfloor$, there would not exist common vectors and there exists internal conflicts within cycle $k$. Otherwise, there would not be internal conflicts within cycle $k$ and there are $\Delta-\left\lfloor l_{k} / 2\right\rfloor$ common precoding vectors shared by every node in cycle $k$. Also note that the column space is preserved by random permutation. Then randomly generate $l_{k}-\Delta+\left(\Delta-\left\lfloor l_{k} / 2\right\rfloor\right)^{+}$vectors, each $(2 \Delta+1) \times 1$, and call them $\mathbf{v}_{\Delta-\left(\Delta-\left\lfloor l_{k} / 2\right\rfloor\right)^{+}+1}^{k}\left(C_{k}\right), \quad \mathbf{v}_{\Delta-\left(\Delta-\left\lfloor l_{k} / 2\right\rfloor\right)^{+}+2}^{k}\left(C_{k}\right), \cdots, \mathbf{v}_{l_{k}}^{k}\left(C_{k}\right)$. Now assign the vectors cyclically (subscripts modulo $l_{k}$ ) for cycle $k$ as shown at the top of the next page, where $\mathbf{U}^{k}\left(C_{k}\right)=\left[\mathbf{u}_{1}^{k}\left(C_{k}\right), \mathbf{u}_{2}^{k}\left(C_{k}\right), \ldots, \mathbf{u}_{\left(\Delta-\left\lfloor l_{k} / 2\right\rfloor\right)^{+}}^{k}\left(C_{k}\right)\right]$ represents all the common vectors. Note that this construction ensures that any adjacent two nodes share $\left(\Delta-\left\lfloor l_{k} / 2\right\rfloor\right)^{+}+\Delta-\left(\Delta-\left\lfloor l_{k} / 2\right\rfloor\right)^{+}-1=\Delta-1$ dimensional precoding space. And message nodes that are connected by a (minimum) path of two edges in the cycle have an overlap of $\Delta-2$ dimensions, message nodes that are connected by a path of three edges have an overlap of $\Delta-3$ dimensions, and so on, so that messages that are connected by a path of $\Delta$ edges (or more) have no overlap. Thus, all conflicts within the cycle (if exist) are avoided. 


$$
\begin{aligned}
& W_{1}\left(C_{k}\right): \mathbf{U}^{k}\left(C_{k}\right), \mathbf{v}_{1}^{k}\left(C_{k}\right), \mathbf{v}_{2}^{k}\left(C_{k}\right), \cdots, \mathbf{v}_{\Delta-\left(\Delta-\left\lfloor l_{k} / 2\right\rfloor\right)^{+}}^{k}\left(C_{k}\right) \\
& W_{2}\left(C_{k}\right): \mathbf{U}^{k}\left(C_{k}\right), \mathbf{v}_{2}^{k}\left(C_{k}\right), \mathbf{v}_{3}^{k}\left(C_{k}\right), \cdots, \mathbf{v}_{\Delta-\left(\Delta-\left\lfloor l_{k} / 2\right\rfloor\right)^{+}+1}^{k}\left(C_{k}\right) \\
& W_{3}\left(C_{k}\right): \mathbf{U}^{k}\left(C_{k}\right), \mathbf{v}_{3}^{k}\left(C_{k}\right), \mathbf{v}_{4}^{k}\left(C_{k}\right), \cdots, \mathbf{v}_{\Delta-\left(\Delta-\left\lfloor l_{k} / 2\right\rfloor\right)^{+}+2}^{k}\left(C_{k}\right) \\
& \text { : : : } \\
& W_{l_{k}}\left(C_{k}\right): \mathbf{U}^{k}\left(C_{k}\right), \mathbf{v}_{l_{k}}^{k}\left(C_{k}\right), \mathbf{v}_{1}^{k}\left(C_{k}\right), \cdots, \mathbf{v}_{\Delta-\left(\Delta-\left\lfloor l_{k} / 2\right\rfloor\right)^{+}-1}^{k}\left(C_{k}\right)
\end{aligned}
$$

3) Now we proceed to all the other nodes that are connected to $W_{1}\left(A_{i}\right)$ but do not form a cycle with $W_{1}\left(A_{i}\right)$. Enqueue all these nodes. For each such node $W_{j}\left(A_{i}\right), j \neq 1$, precoding matrix is generated as:

$$
\begin{aligned}
& \mathbf{Q}_{j}\left(A_{i}\right)=\operatorname{rand}(\Delta, \Delta-1) \\
& \mathbf{V}_{j}\left(A_{i}\right)=\left[\mathbf{V}_{1}\left(A_{i}\right) \mathbf{Q}_{j}\left(A_{i}\right) \quad \operatorname{rand}(2 \Delta+1,1)\right]
\end{aligned}
$$

The random matrix $\mathbf{Q}_{j}\left(A_{i}\right)$ is simply meant to choose a generic $\Delta-1$ dimensional subspace from $\mathbf{V}_{1}\left(A_{i}\right)$. This is appended with an independently generated vector that will (with high probability over a sufficiently large field) be in general position and linear independent with respect to $\mathbf{V}_{1}\left(A_{i}\right) \mathbf{Q}_{j}\left(A_{i}\right)$. Thus, $\mathbf{V}_{1}\left(A_{i}\right)$ and $\mathbf{V}_{j}\left(A_{i}\right)$ are connected by an edge in the alignment graph and have a $\Delta-1$ dimensional overlap between their signal spaces.

4) Operations related to $W_{1}\left(A_{i}\right)$ are done. Dequeue and consider the next message node $W_{j}\left(A_{i}\right)$ in the queue. Now $W_{j}\left(A_{i}\right)$ becomes the new center node and go back to step 2 to deal with all the unassigned nodes. When the queue is empty again, the precoding matrix assignment is completed. Since the alignment graph does not have overlapping cycles, messages that are connected by a path (may cross multiple cycles) of $\Delta$ edges (or more) have no overlap with such construction as common vectors within different cycles are in generic positions because of the random permutation and other new appended vectors are generic naturally.

After completing the construction, we are left to show that with high probability the desired signals at each destination have no overlap with the interference. Without loss of generality we will assume each destination desires one message.

Consider a destination whose interfering messages come from an alignment set that has no internal conflicts. Thus, all interfering messages span the same $\Delta$ dimensional space, and the desired signal (because it belongs to a different alignment set) spans an independently generated $\Delta$ dimensional space. Since the overall number of dimensions is $2 \Delta+1$, with high probability these two spaces have no overlap.

Henceforth we consider only destinations whose interfering messages come from an alignment set that has internal conflicts.

Suppose the desired message, say $W_{i}$, sees two interferers $W_{j}, W_{k}$. Then $W_{j}, W_{k}$ must be connected by an edge in the alignment graph. Therefore, they must have a $\Delta-1$ dimensional overlap whether they are a part of a cycle or not, so that together they must span $\Delta+\Delta-(\Delta-1)=\Delta+1$ dimensions. Further, if $W_{i}$ is in the same alignment set, then $W_{j}, W_{k}$ must be at least $\Delta$ edges away from $W_{i}$, so that with high probability the union of the spans of $\mathbf{V}_{j}, \mathbf{V}_{k}$ is in general position with respect to $\mathbf{V}_{i}$. Since the total space is $2 \Delta+1$ dimensional, it is big enough to accommodate the interference and the desired signal without forcing them to overlap. Thus, the desired signal does not overlap with interference with high probability. If the message $W_{i}$ is in a different alignment set then again its signal space is independently generated and with high probability has no overlap with the space spanned by the interference. If the message $W_{i}$ sees only one interferer, $W_{j}$, then once again because $W_{i}, W_{j}$ are at least $\Delta$ edges apart (or belong to different alignment sets), the signal spaces $\mathbf{V}_{i}, \mathbf{V}_{j}$ have no overlap with high probability.

\section{APPENDIX}

The index coding problem consists of $S$ source nodes, labeled $S_{j}, j \in\{1,2, \ldots, S\}, D$ destination nodes, labeled $D_{i}, i \in\{1,2, \ldots, D\}$ and two additional nodes, labeled as $N_{1}, N_{2}$, that are connected by a unit capacity edge going from $N_{1}$ to $N_{2}$, known as the bottleneck link. There is an infinite capacity link from every source to the node $N_{1}$, and an infinite capacity link from $N_{2}$ to every destination node. What it means is simply that $N_{1}$ knows all the messages, so all the coding is performed at $N_{1}$, and the output of the bottleneck link is available to all destination nodes.

Source node $S_{j}$ has a set of independent messages, $\mathcal{W}\left(S_{j}\right)$, that it wants to send to their desired destinations. Destination node $D_{i}$ has a set of independent messages $\mathcal{W}\left(D_{i}\right)$ that it desires. We distinguish between multiple groupcast settings where each message can be desired by multiple destinations and multiple unicast settings where each message cannot be desired by more than one destination. The side information structure is defined by the antidote matrix $\mathcal{A}=\left[a_{i j}\right]_{D \times S}$ of zeros and ones where $a_{i j}=1$ means a direct link of infinite capacity exists from $S_{j}$ to $D_{i}$ and provides $\mathcal{W}\left(S_{j}\right)$ to $D_{i}$, otherwise $a_{i j}=0$ and no path exists from $S_{j}$ to $D_{i}$ except through the bottleneck link. To avoid degenerate cases, we assume desired message are not available as side information and must pass through the bottleneck link, i.e., $a_{i j}=0$ whenever $\mathcal{W}\left(D_{i}\right) \cap \mathcal{W}\left(S_{j}\right) \neq \phi$. The bottleneck link is able to transmit one symbol from $\mathbb{G F}$ each channel use and the symbol transmitted is denoted as $\mathcal{S}$.

Coding schemes, probability of error, achievable rates and capacity region are defined in the standard information theoretic sense of vanishing probability of error. We are interested in the symmetric capacity $R$ normalized by the capacity 
of the bottleneck link, $\log (|\mathbb{G} \mathbb{F}|)$. Although the choice of field is irrelevant to the normalized capacity of the index coding problem, we assume the field is large to simplify the design of achievable scheme. Throughout the paper, we will use the notion of linear schemes defined in detail in [3, Appendix A]. Linear capacity is defined similar to capacity, but with the constraint that coding schemes must be linear.

We proceed to the definition of alignment and conflict graphs for the index coding problem.

1) Alignment Graph: Messages $W_{i}$ and $W_{j}$ are connected with a solid black edge if the source(s) of both these messages are not available as antidotes to a destination that desires message $W_{k} \notin\left\{W_{i}, W_{j}\right\}$.

2) Conflict Graph: Each message $W_{i}$ is connected by a dashed red edge to all other messages whose sources are are not available as antidotes to a destination that desires message $W_{i}$.

Also let us define Alignment Set to be each connected component (through solid black edges) of an alignment graph and Internal Conflict which refers to the occasion where two messages that belong to the same alignment set have a conflict (dashed red) edge between them. For two messages that have an internal conflict between them, the Conflict Distance is defined as the minimum number of alignment graph edges that need to be traversed to go from one node to the other. The minimum conflict distance of all internal conflicts is denoted as $\Delta$. If message $W_{j}$ is not available as antidote to destination $i$, we also call $W_{j}$ interference for destination $i$. $W_{i}, W_{j}$ is abbreviated as $W_{i, j}$, etc. $W_{i, j}^{c}$ is used to denote all the messages except $W_{i, j}$.

Finally, we say that the alignment graph has non-overlapping cycles when no edge is a part of two cycles in the graph.

\section{REFERENCES}

[1] Y. Birk and T. Kol, "Informed-source coding-on-demand (ISCOD) over broadcast channels," in Proc. 17th Annu. Joint Conf. IEEE Comput. Commun. Soc. (INFOCOM), vol. 3. Mar./Apr. 1998 , pp. $1257-1264$.

[2] S. A. Jafar, "Interference alignment-A new look at signal dimensions in a communication network," Found. Trends Commun. Inf. Theory, vol. 7, no. 1, pp. 1-134, 2011.

[3] S. A. Jafar, "Topological interference management through index coding," IEEE Trans. Inf. Theory, vol. 60, no. 1, pp. 529-568, Jan. 2014.

[4] H. Maleki, V. Cadambe, and S. A. Jafar, "Index coding: An interference alignment perspective," in Proc. IEEE ISIT, Jul. 2012, pp. 2236-2240. [Online]. Available: http://arxiv.org/abs/1205.1483

[5] S. El Rouayheb, A. Sprintson, and C. Georghiades, "On the index coding problem and its relation to network coding and matroid theory," IEEE Trans. Inf. Theory, vol. 56, no. 7, pp. 3187-3195, Jul. 2010.

[6] M. Effros, S. El Rouayheb, and M. Langberg. (Nov. 2012). "An equivalence between network coding and index coding." [Online]. Available: http://arxiv.org/abs/1211.6660

[7] Z. Bar-Yossef, Y. Birk, T. S. Jayram, and T. Kol, "Index coding with side information," IEEE Trans. Inf. Theory, vol. 57, no. 3, pp. 1479-1494, Mar. 2011.

[8] A. Blasiak, R. Kleinberg, and E. Lubetzky, "Broadcasting with side information: Bounding and approximating the broadcast rate," IEEE Trans. Inf. Theory, vol. 59, no. 9, pp. 5811-5823, 2013.

[9] A. S. Tehrani, A. G. Dimakis, and M. J. Neely, "Bipartite index coding," in Proc. IEEE Int. Symp. Inf. Theory (ISIT), Jul. 2012, pp. 2246-2250.
[10] K. Shanmugam, A. G. Dimakis, and M. Langberg. (Jan. 2013). "Local graph coloring and index coding." [Online]. Available: http://arxiv.org/abs/1301.5359

[11] N. Alon, A. Hasidim, E. Lubetzky, U. Stav, and A. Weinstein (Jun. 2008). "Broadcasting with side information." [Online]. Available: http://arxiv.org/pdf/0806.3246v1.pdf

[12] S. A. Jafar. (Jan. 2013). "Topological interference management through index coding." [Online]. Available: http://arxiv.org/abs/1301.3106

[13] A. Blasiak, R. Kleinberg, and E. Lubetzky. (Aug. 2011). "Lexicographic products and the power of non-linear network coding." [Online] Available: http://arxiv.org/abs/1108.2489

[14] F. Arbabjolfaei, B. Bandemer, Y.-H. Kim, E. Sasoglu, and L. Wang. (Feb. 2013). "On the capacity region for index coding." [Online] Available: http://arxiv.org/abs/1302.1601v1

[15] N. Naderializadeh and A. S. Avestimehr. (2013). "Interference networks with no CSIT: Impact of topology." [Online]. Available: http://arxiv.org/abs/1302.0296

[16] R. W. Yeung, Information Theory and Network Coding. New York, NY, USA: Springer-Verlag, 2008.

[17] D. Hammer, A. Romashchenko, A. Shen, and N. Vereshchagin, "Inequalities for Shannon entropy and Kolmogorov complexity," J. Comput. Syst. Sci., vol. 60, no. 2, pp. 442-464, 2000.

[18] Z. Zhang and R. W. Yeung, "On characterization of entropy function via information inequalities," IEEE Trans. Inf. Theory, vol. 44, no. 4, pp. 1440-1452, Jul. 1998.

[19] K. Makarychev, Y. Makarychev, A. Romashchenko, an N. Vereshchagin, "A new class of non-Shannon-type inequalities for entropies," Commun. Inf. Syst., vol. 2, no. 2, pp. 147-166, Dec. 2002.

[20] F. Matus, "Infinitely many information inequalities," in Proc. Int. Symp. Inf. Theory (ISIT), Jun. 2007, pp. 41-44.

[21] R. Dougherty, C. Freiling, and K. Zeger. (Apr. 2011). "Non-Shannon information inequalities in four random variables." [Online]. Available: http://arxiv.org/abs/1104.3602v1

[22] A. W. Ingleton, "Representation of matroids," Combinat. Math. Appl., vol. 44, pp. 149-167, Jul. 1971.

[23] R. Kinser, "New inequalities for subspace arrangements," J. Combinat. Theory, A, vol. 118, no. 1, pp. 152-161, Jan. 2011.

[24] R. Dougherty, C. Freiling, and K. Zeger. (Jul. 2010). "Linear rank inequalities on five or more variables." [Online]. Available: http://arxiv.org/abs/0910.0284v3

[25] R. Dougherty, C. Freiling, and K. Zeger, "Network coding and matroid theory," Proc. IEEE, vol. 99, no. 3, pp. 388-405, Mar. 2011.

[26] T. H. Chan and A. Grant, "Dualities between entropy functions and network codes," IEEE Trans. Inf. Theory, vol. 54, no. 10, pp. 4470-4487, Oct. 2008.

[27] R. Dougherty, C. Freiling, and K. Zeger, "Networks, matroids, and non-Shannon information inequalities," IEEE Trans. Inf. Theory, vol. 53, no. 6, pp. 1949-1969, Jun. 2007.

[28] R. W. Yeung, "Facets of entropy," IEEE Inf. Theory Soc. Newslett., vol. 62, no. 8, pp. 6-16, Dec. 2012.

[29] T. Chan, "Recent progresses in characterising information inequalities," Entropy, vol. 13, no. 2, pp. 379-401, 2011.

[30] R. Baber, D. Christofides, A. N. Dang, S. Riis, and E. R. Vaughan, "Multiple unicasts, graph guessing games, and non-Shannon inequalities," in Proc. Int. Symp. NetCod, Jun. 2013, pp. 1-6.

[31] J. G. Oxley, Matroid Theory. New York, NY, USA: Oxford Univ. Press, 1992.

Hua Sun (S'12) received the B.E. degree in Communications Engineering from Beijing University of Posts and Telecommunications, Beijing, China, in 2011, the M.S. degree in Electrical and Computer Engineering from University of California Irvine, USA, in 2013. He is currently pursuing the $\mathrm{Ph} . \mathrm{D}$. degree at the University of California Irvine. His research interests include multiuser information theory and wireless communications. 
Syed Ali Jafar (S'99-M'04-SM'09-F'14) received his B. Tech. from IIT Delhi, India, in 1997, M.S. from Caltech, USA, in 1999, and Ph.D. from Stanford, USA, in 2003, all in Electrical Engineering. His industry experience includes positions at Lucent Bell Labs, Qualcomm Inc. and Hughes Software Systems. He is a Professor in the Department of Electrical Engineering and Computer Science at the University of California Irvine, Irvine, CA USA. His research interests include multiuser information theory, wireless communications and network coding.

Dr. Jafar received the NSF CAREER award in 2006, the ONR Young Investigator Award in 2008, and the School of Engineering Maseeh Outstanding Research Award in 2010, his co-authored papers received Best Journal Paper Awards from the IEEE Information Theory Society in 2009, from the IEEE Communication Society in 2013, and from the IEEE Signal Processing Society in 2015, and Best Conference Paper Awards at
IEEE GLOBECOM 2012 and at IEEE GLOBECOM 2014. Dr. Jafar received the UC Irvine EECS Professor of the Year award five times, in 2006, 2009, 2011, 2012, and 2014, from the Engineering Students Council and the Teaching Excellence Award in 2012 from the School of Engineering. He was a University of Canterbury Erskine Fellow in 2010 and an IEEE Communications Society Distinguished Lecturer for 2013-2014. Dr. Jafar was recognized as a Thomson Reuters Highly Cited Researcher and included by Sciencewatch among The Worlds Most Influential Scientific Minds in 2014. He served as Associate Editor for IEEE TRANSACTIONS ON COMMUNiCATIONS 2004-2009, for IEEE COMMUNICATIONS LETTERS 2008-2009 and for IEEE TRANSACTIONS ON INFORMATION THEORY 2009-2012. Dr. Jafar was elevated to IEEE Fellow, Class of 2014, for contributions to analyzing the capacity of wireless communication networks. 